\title{
Kognitive und kulturelle Aspekte des Übersetzens
}

\author{
Gerd Wotjak ${ }^{1}$
}

\begin{abstract}
This paper investigates the interrelation between cultural, cognitive and communicative-linguistic phenomena. Cultural aspects not only mark encyclopaedic knowledge but they also influence language as a system and language use. Cultural knowledge manifests itself in the meaning of certain lexemes and in culturally marked representations of world knowledge, as knowledge of action and stereotyped behaviour. Cultural knowledge also influences the preferences of speakers when producing texts, i.e. the selection, organization and combination of lexical and morpho-syntactical elements. The resulting difficulties for translation are thoroughly discussed.
\end{abstract}

Keywords: science of translation; culture and language; cultural knowledge; Spanish; German.

Zusammenfassung: In dieser Arbeit wird der Interrelation zwischen kulturellen, kognitiven und kommunikativ-sprachlichen Phänomenen nachgegangen. Kulturelles prägt nicht nur das enzyklopädische Weltwissen, sondern beeinflusst auch die Sprache als System und den Sprachgebrauch. Kulturelles Wissen manifestiert sich in den Bedeutungen bestimmter Lexeme, in kulturgeprägten Weltwissensrepräsentationen, als Handlungsmusterwissen und Verhaltensstereotypkenntnis, sowie in Präferenzen für die Selektion, Anordnung und Kombination von sprachlichen Systemelementen lexikalischer wie morphosyntaktischer Art zu Textsortenexemplaren. Die der Übersetzungstätigkeit daraus erwachsenden Schwierigkeiten werden differenziert durchleuchtet.

Stichwörter: Translationswissenschaft; Kultur und Sprache; Kulturelles Wissen; Spanisch; Deutsch.

0. Im Zusammenhang mit der verstärkten internationalen Kooperation und der Globalisierung des Austausches von Waren, Dienstleistungen und Ideen ist neben der zunehmenden Verbreitung des Englischen als lingua franca auch eine deutliche Zunahme der translatorischen Aktivitäten (Dolmetsch- wie Übersetzungsleistungen) zu verzeichnen. Auf diese gestiegenen Anforderungen haben zunächst einige wenige, inzwischen aber weltweit deutlich über 200 zumeist universitäre Ausbildungseinrichtungen neben Fach(hoch)schulen und privaten Instituten reagiert Tendenz steigend, verspricht man sich doch gute Einsatzmöglichkeiten für die professionellen Übersetzer, auch über den engeren Einsatzbereich des Übersetzens hinaus etwa im Language Engineering, bspw. in der Softwarelokalisation oder der Terminographie/ der Erstellung und Pflege elektronischer Datenbanken, etc.

\footnotetext{
${ }^{1}$ Emeritierter Professor des IALT, Universität Leipzig. E-Mail: wotjak@uni-leipzig.de
} 
0.1 Theoretische Überlegungen zu den in hohem Maße komplexen Vorgängen beim Übersetzen/Dolmetschen, d.h. bei der Translation, finden sich zwar auch schon in weit zurückliegenden Jahrhunderten, nicht zufällig aus der Feder bekannter Übersetzer selbst (bspw. Hieronymus, Luther, Schleiermacher) und zumeist als eine Art Rechtfertigung ihres Vorgehens und Darlegung ihres translatorischen Credos.

Die Entstehung der Translatologie/ Translation Studies, Übersetzungswissenschaft, traductologie oder Translemik jedoch als wissenschaftliche Beschäftigung im engeren Sinne mit einem deutlich ausgeweiteten Untersuchungsgegenstand und Anspruch ${ }^{2}$ datiert faktisch erst vom Ende der 50er und Mitte der 60er Jahre des XX. Jahrhunderts (dazu eine gute Zusammenschau in SIEVER 2008; vgl. auch HurTAdo AlbiR 2001).

0.2 Bei der Translatologie handelt es sich nicht nur um eine junge Disziplin, die noch immer um ihre Gleichberechtigung, ja sogar um die Anerkennung als eigenständige Disziplin und wichtiges Komplement zur translatorischen Fertigkeitsentwicklung ringen muss. Die Translatologie hat vielmehr gegenstandsbedingt - es geht um die komplexe Interrelation von zweisprachig vermittelter inter- bzw. transkultureller Kommunikation, mit dem Ziel der Vermittlung von Wissen, Werten/Einstellungen, von in hohem Maße kulturgeprägten Sichtweisen auf die Welt über Länder- und Kulturgrenzen hinweg den Charakter einer science-pilote im Sinne COSERIUS 1977, einer den Beitrag mehrerer Einzeldisziplinen erfordernden integrativ-modularen Interdisziplin (dazu SNELLHORNBY 1994; ZyBATOw 2002), was zugleich ihren Reiz wie ihre Crux ausmacht.

0.3 Es kann in diesem Kontext nicht verwundern, wenn das Übersetzen immer mehr Interessenten anzieht, sich andererseits aber mehrere konkurrierende Schulen herausgebildet haben und selbst hinsichtlich grundlegender Aspekte im Einzelnen durchaus abweichende Auffassungen verkündet werden. So tief die Gräben bspw. zwischen Leipziger Übersetzungswissenschaftlicher Schule (Jung 2000; WOTJAK 1995; 1997a; 2003; 2005b) und der Allgemeinen Funktionalen Übersetzungswissenschaft im Gefolge von Reiß/VERMEer 1984, HoltZ-MäNTTÄRi 1984 und NorD (1988, 2002a) auch sein mögen, wobei hier durchaus auch einseitige Verabsolutierungen zu bemerken sind (vgl. WOTJAK 1997a, 2003), so stimmen diese beiden Schulen zusammen mit der

\footnotetext{
${ }^{2}$ Zunächst dominierten religiöse, philosophische und literarische Übersetzungen; erst im 20. Jahrhundert wuchs der Übersetzungsbedarf an naturwissenschaftlich-technischen Texten sprunghaft.
} 
Manipulation School (u.a. LEFEVERE 1996), der Deskriptiven Translatologie (TOURY 1995) und der Pariser Übersetzungswissenschaftlichen Schule (SELESKOVITCH/LEDERER 1984) u.a. dahin gehend überein, dass eine Beschreibung des Übersetzens sich bei weitem nicht in einer linguistisch-semiotischen Beschreibung erschöpfen kann, sondern darüber hinausgehende Überlegungen zu kognitiven wie insbesondere kulturellen Aspekten (neben bzw. auch komplementär zueinander und etwa auch zu kommunikativen Phänomenen) erfordert (dazu u .a. WITTE 2000; CAMPOS PlazA/Ortega ARJONilla 2005). Zwar haben die Leipziger Hauptvertreter Jäger, Kade und Neubert in unterschiedlicher Gewichtung stets eine kommunikationswissenschaftlich-linguistische Herangehensweise präferiert, doch finden sich auch hier z. T. schon 1965 kognitive wie pragmatisch-kulturelle Aspekte thematisiert (NEUBERT 1968; WOTJAK 1991a; 1997a; 2003).

0.4 Inzwischen scheint sich verbreitet eine Definition des Übersetzens als Kulturtransfer bzw. als "Kulturmittlung“ (vgl. dazu u. a. WiTTE 2000; WOTJAK 1993a, b; 2006b) durchgesetzt zu haben. Dabei ist eine solche Akzentuierung allerdings nicht frei von der Gefahr, dass darüber bspw. kognitive Aspekte, aber eben auch kommunikativlinguistisch-semiotische Aspekte der Translation allzu stark vernachlässigt werden. Schon längst ist man von einer im Übrigen nur sehr vereinzelt vertretenen simplifizierenden Ansicht abgerückt, dass man das Übersetzen im Sinne von Karl Krauss etwa als $\ddot{U} b^{\prime}$ ersetzen auffassen könnte. Aber auch an dem auf eine Feststellung von J. Grimm zurückgehenden Bild vom traducere navem, dem Übersetzen als übersetzen von einem Gestade an ein anderes, wird bspw. von HöNIG (1995) Kritik geübt, wobei nach seiner Überzeugung dieses Bild durch das adäquatere eines Brückenschlagens ersetzt worden sollte.

1. Ohne hier eine kritische Würdigung der im Einzelnen vorgenommenen Beschreibungsversuche anstreben zu können (vgl. dazu insbesondere JuNG 2000, SALEVSKy 2002; SNELl-HoRnBy ET AL. 1998; Koller 1992; G.WotJAK 2006; SieVeR 2008), sei immerhin darauf verwiesen, dass bspw. schon durch G. MouniN (1963) und E.A.NIDA (1964) mehr oder weniger detailliert auf das soziokulturelle Bedingungsgefüge für die Translation eingegangen wurde und sich selbst bei CATFORD 
(1965) mit dem postulierten Ersetzen eines Textes in einer Situation/ einem Kontext eine mögliche Relation zu soziokulturellen Faktoren herstellen lässt.

Schon früh wurde mit dem Interesse an dem sprachlichen Relativismus/ dem linguistischen Weltanschauungsproblem als einem immer wieder bemühten Haupthindernis für die Übersetzbarkeit ${ }^{3}$ im Umfeld translatologischer Überlegungen auch Fragen der Beziehung von Sprache und Denken/Kognition thematisiert. Hinzu kommt, dass eine noch so oberflächliche Beobachtung der Phänomene der übersetzerischen Tätigkeit offenbart, dass der Translator als Quasiempfänger des Ausgangstextes $/ \mathrm{T}_{\mathrm{AS}}$ in einer semasiologischen Analysephase die Mitteilung/Botschaft/Message bzw. den kommunikativen Sinn (dazu WOTJAK 2005b) erschließen/dekodieren und dann als Sender in einem onomasiologischen Herangehen in der Synthesephase diesen Sinn (dazu aus der Sicht der Pariser Schule LeDERER 1994; Seleskovitch/Lederer 1984; Hurtado Albir 2001; SÁez Hermosilla 1994) neu vertexten/ enkodieren muss, also immer neben linguistisch-semiotischen Phänomenen kognitive Aspekte untrennbar mit kommunikativen verwoben und grundlegend fürs Übersetzen sind (vgl. dazu die Darstellungen bei KADE 1968 unter (1)).

\section{Sender/S $\mathrm{S}_{\mathrm{AS}} \rightarrow \mathrm{i} \sim \mathrm{e}$ Translator/T $\sim \mathrm{i}^{\prime} \sim \mathrm{e}^{\prime}$ Empfänger/E $\mathrm{E}_{\mathrm{ZS}}$}

Legende: AS-Sender produziert eine Intention/intendierten Effekt i, den der als Quasiempfänger fungierende Translator als realisierten Effekt e dekodiert; dann fungiert T als Quasisender und produziert einen intendierten Effekt $\mathbf{i}^{\prime}$, der schon nicht mehr völlig identisch mit $\mathbf{i}$ ist, dieser $\mathbf{i}^{\prime}$ wird wiederum von den Empfängern in ZS als $\mathbf{e}^{\prime}$ aktualisiert, der dem ursprünglichen realisierten Effekt e des $\mathrm{T}$ nur angenähert ist.

1.1 Dass in der translatologischen Forschung der 60er und 70er Jahre, vor allem, aber nicht ausschließlich in der Leipziger Schule, die Betonung kultureller wie etwa auch außersprachlich-pragmatischer und selbst kognitiver Faktoren deutlich überlagert wurde durch eine vor allem linguistisch basierte Beschreibung der kommunikativen Äquivalenz von Texten ist nicht zuletzt wohl auch dem vorherrschenden Trend geschuldet, demzufolge ein einer wissenschaftlich exakten Beschreibung angemessenes Arsenal von Postulaten, Hypothesen und Methoden damals noch am ehesten aus der

\footnotetext{
${ }^{3}$ Vgl. dazu u. a. schon Humboldt im 19. Jahrhundert; dann Sapir/Whorf, aber bspw. auch CARY 1960. Eine detaillierte Auseinandersetzung dazu findet sich u. a. schon bei MOUNIN 1963; vgl. auch KADE 1971.
} 
Linguistik entlehnt werden konnte. Es kann nicht verwundern, dass dabei ein weitgehend mit Rekurs auf linguistische Mittel und Beschreibungsverfahren machbarer Vergleich von Original und Translat als Produkt der übersetzerischen Tätigkeit bzw. Handlung vorgezogen wurde einer wohl nur durch Rekurs auf psycholinguistische Methoden leistbaren Beschreibung des Übersetzens als Prozess, dessen wesentliche Teile sich in der einer direkten Beobachtung nicht zugänglichen Black Box des Gehirns des Translators (vgl. aber KRINGS 1986) abspielen.

Wiewohl von Anfang an der Text als die letztendliche Übersetzungseinheit postuliert und damit durchaus der Ausweitung linguistischer Analysen auf die Performanz/Verwendung/Rede Rechnung getragen wurde in einer Zeit dominanter Akzentuierung der Kompetenz etwa seitens der Mainstreamlinguistik der Generativen Transformationsgrammatik Chomskys, nahmen die frühen Versuche translatologischer Beschreibungen nicht zufällig besonders auf subtextuelle Übersetzungseinheiten über Satz bis hin zum Wort Bezug. Immerhin stand in den 60er Jahren noch keine textlinguistische oder gar allgemeiner textwissenschaftliche Methodologie zur Verfügung, deren Ergebnisse dann bspw. von Neubert (1985) und NeuberT/ SHREve (1992) in ihrem Bezug auf die Translation einbezogen, aber bspw. auch von REIß (1971) schon mit Bezug auf Textsortenbeschreibungen und in REIß/VERMEER (1996) vertieft angesprochen wurden.

Es verdient in diesem Kontext Beachtung, dass die Leipziger Übersetzungswissenschaft mit ihrer linguistischen Fundierung sich sehr früh mit der insgesamt eher vernachlässigten und auch heute noch virulenten Problematik der Bedeutung und im Zusammenhang mit der Bestimmung der kommunikativen (zunächst konkurrierend auch funktionalen) Äquivalenz auch ausdrücklich mit kognitiven Aspekten, etwa der postulierten weitgehenden Deckungsgleichheit der durch den Text in den AS- bzw. ZSRezipienten koaktivierten Bewusstseinskomplexe, auseinandersetzte. So nimmt es auch nicht wunder, dass sich in Jägers Translationslinguistik von 1975 mehrfache detaillierte Bezüge auf die Bedeutungsproblematik, auf den Text und insbesondere erste Versuche einer die Textsemantik, insbesondere einer die aktuelle Gliederung mit einbeziehenden Beschreibung, finden ${ }^{4}$.

\footnotetext{
${ }^{4}$ Vgl. dazu vertiefend u. a. JÄGER 1986, WOTJAK 1986, 1997b; 2006a.
} 
1.2 Mit der Einbeziehung der Verstehensvoraussetzungen durch JÄGER/MÜLLER (1982) (vgl. davor schon BASTIAN 1974, 1979) schließlich wurden unterschiedliche Aspekte der translatorischen Kompetenz, des durch den Translator zu beachtenden, nicht selten kulturgeprägt divergierenden Wissensbesitzes der AS- und ZS-Rezipienten, und damit neben Kulturellem ebenfalls Kognitives explizit angesprochen. In diesem Kontext könnte nachstehende Auflistung von durch den Translator wie den Originalsender und die Rezipienten von $\mathrm{T}_{\mathrm{AS}}$ und Translat $\mathrm{zu}$ berücksichtigenden Unterarten von Teilwissensdomänen (vgl. WOTJAK 2001; 2005a) einen ungefähren Eindruck von der Komplexität der Übersetzungshandlung wie der Vielfalt von Wissens- und Fertigkeitskomponenten in der translatorischen Kompetenz (dazu u.a. RISKU 1998) vermitteln.

Hinsichtlich des Wissens müsste wohl zwischen dem interiorisiert verfügbaren bzw. zur Durchführung des Translationsaktes unvermeidbar $\mathrm{zu}$ erwerbenden generischen, insbesondere themen- bzw. kommunikationsgegenstandsbezogenen, enzyklopädischen Wissen (dazu B. NORD 2002) einerseits und dem episodisch ad hoc sowie in der Äußerungsdeixis Ego-Hic-Nunc (dazu besonders WANDRUSZKA 1969 unter Bezug auf BÜHLER 1934) aus der kommunikativen Begleitsituation und dem koaktivierten so genannten soziokulturellen Hintergrundwissen durch die Rezipienten, darunter den Translator, im Hinblick auf das Original zu entnehmendem Situations- oder Kontextwissen andererseits unterschieden werden. Während letzteres in gewissem Sinne unikales Wissen um das Wann, Wo, durch Wen (Autor) und für Wen (Adressat), in welcher konkreten sozialen Interaktionsbeziehung zum Sender bzw. in welchem sozial-kommunikativen wie kulturellen Bedingungsgefüge umfasst, wobei letztere Komponenten in der Regel das Einmalige transzendieren, zählen zum enzyklopädischen Wissensbesitz als über ein n-Tupel von vorgängigen Erkenntnis- wie Kommunikationsakten erworbenem "Langzeitwissen” u. a. nachstehende Teilbereiche (vgl. (2) - Aufzählung ohne Wertung und Beachtung der vorliegenden Interdependenzen): 
(2) Wissensdomänen

\section{enzyklopädisches Wissen O’enz}

\section{A}

Subdomänen von O'enz ohne Bezug auf den

Menschen

Naturwissenschaften

O'phys O'math

$\mathrm{O}^{\prime}$ chem $\mathrm{O}^{\prime} \log$...
Wissensdomänen mit Bezug auf das Inviduum

Sciences de l’homme

O’bio O'med O'psych

O’physio O'geo ... soziales Interaktionswissen $\mathrm{O}^{\prime}$ soz

Mensch als soziales Wesen

B $\rightarrow$ Gesellschaftswissenschaften:

O’hist O'polit O'ök O’soz O'philos

O’kult O’komm

O’semiot O’ling

O’lex O’synt O’morphol...

A naturwissenschaftlich-technische Wissensdomänen, etwa O'math, O’bio; O'chem; O'physik; O’med; O'techn (mit Subspezifikationen, etwa KFZ-Technik);

B geisteswissenschaftliche Wissensdomänen bzw. durch diese Wissenschaften beschriebene Sachverhaltsdomänen: O’soz als soziale Interaktion; O'phil; O’hist; aber bspw. auch als im weiteren Sinne Wissen über operativ-kommunikativen Umgang mit den erwähnten Wissensteilbereichen als O’komm mit der Unterkomponente O’ling, die eine notwendige Brücken/Mittlerfunktion zwischen materialisierten Kommunikationsressourcen par excellence und den durch diese "transportierten” Konzeptualisierungen/Konzepten der unterschiedlichsten Teilwissensdomänen (einschließlich von selbstreflexiven linguistischen Konzeptbildungen) wahrnimmt.

Dabei werden nachfolgende Überlegungen zur Repräsentation von kulturellen Faktoren deren mannigfache Einwirkung auf bzw. Wechselwirkung mit verschiedenen Teilwissensbereichen verdeutlichen; es besteht aber auch kein Zweifel daran, dass eine wichtige Schaltstelle zu wesentlichen Teilbereichen kultureller Designatsdomänen in den Bedeutungen lexikalischer Einheiten liegt (hier sind auch die Konzeptualisierungen der anderen Wissensbereiche verschaltet), darüber hinaus aber bspw. auch in den Textsortenkonventionen wie in Präferenzen für den Gebrauch systemhaft disponibler Ressourcen, darunter von Sprechakten und Kommunikationsstrategien, seinen Niederschlag gefunden hat (dazu u. a. Lorenz/WotJAK 1977; WotJAK 2006a).

1.3 Halten wir als vorläufiges erstes Fazit im Hinblick auf die uns interessierende Fragestellung aus dem gerafft-selektiven Rückblick auf die Anfänge translatologischer Untersuchungen fest: 
1.3.1 Übersetzen ist kein üb'ersetzen; zumindest nicht in dem üblichen, zudem stark vereinfachenden Verständnis: denn zumindest wäre eine Ersetzung des $T_{\mathrm{AS}}$ durch einen $\mathrm{T}_{\mathrm{ZS}}$ an die Bedingung geknüpft, dass das Translat gegenüber dem Original als kommunikativ äquivalent und zudem auch als kommunikativ angemessen, d.h. den ZSTextsortenkonventionen entsprechend (vgl. dazu später) gestaltet, sein müsste. Das gewählte Bild aber suggeriert doch eher, dass es möglich und angeraten sein könnte, für AS-Textelemente, etwa sogar Wörter, einfach ZS-Entsprechungen zu wählen. Wird dagegen die obige Interpretation präferiert, so könnte der Gedanke aufkommen, dass der zu substituierende $\mathrm{T}_{\mathrm{Zs}}$ in toto präexistent ist und als solcher, etwa als komplett zur Verfügung stehender Paralleltext nur aus einem Inventar der unterschiedlichsten Paralleltexte ausgewählt werden und nicht durch den Translator erst produziert werden müsste.

1.3.2 Auch das dem Übersetzen, traducere navem, vorgezogene Bild des Brückenschlags (HÖNIG 1995) hat seine interpretatorischen Tücken; gewiss stellen bereits vorgelegte Übersetzungen Brücken für eine interkulturelle Kommunikation dar und erleichtern nachfolgende Übersetzungsarbeiten. Doch werden durch eine Übersetzung zwar Kultur- wie Sprachbarrieren mittels dieser Brücke überspannt, doch vollzieht sich beim translatorischen Brückenbau wohl zugleich auch ein bestimmter Informationstransfer, wird also eine Botschaft des Originals einem ZS-Rezipientenkreis zum verstehenden Deuten zusammen mit dem Brückenbau näher gebracht (auf die Problematik solcher metaphorischer Benennungen macht insbesondere MARTíN DE LEÓN (2005) aufmerksam).

1.3.3 Übersetzen ist aber bei aller insbesondere durch die kulturelle Wende in der Translatologie betonten Kulturgeprägtheit wohl doch kein Kulturtransfer, zumindest nicht im landläufigen Sinne von Transfer, da ja durchaus nicht immer Aspekte der ASKultur, die in der AS-Diskurswelt präsent sind und bspw. auch deren formal-stilistische Präsentation gemäß bestimmten Textsortenkonventionen beeinflussen, in das ZSTranslat transferiert, hinübertransportiert, übergesetzt, werden. Hier schiene es in nicht wenigen Fällen adäquater, würde von einem Ersatz von AS-Kulturgeprägtheit durch die kommunikativ-situativ angemessenen ZS-Kulturcharakteristika gesprochen. 
1.3.4 Wird Übersetzen als zweisprachig vermittelte, Kulturgrenzen überschreitende (transkulturelle) Kommunikation verstanden, so könnte damit eine viel versprechende Ausgangsbasis für weiterführende Überlegungen gegeben sein. Dabei ergibt sich bei einer solchen Sichtweise, wie sie mit Einschränkungen durch die Leipziger Übersetzungswissenschaft vertreten wurde, nicht automatisch eine Überbetonung linguistischer Aspekte, noch führt sie automatisch zur Akzeptanz der Konzepte der kommunikativen Äquivalenz und kommunikativen Angemessenheit, wiewohl uns beide im Verband nach wie vor essentiell für eine wie auch immer bestimmte translatologische Untersuchung erscheinen. Zwar setzt interpersonale Kommunikation die Benutzung vor allem sprachlicher Mittel voraus, doch wird damit die Verwendung weiterer Zeichen ebenso wenig ausgeschlossen wie die von paralinguistischen Mitteln wie Gestik und Mimik, die allerdings wohl nur beim Dolmetschen direkt translatologisch relevant sind.

Da Kommunikation als entscheidendes Unterpfand und Prämisse als Resultat sozialer Interaktion ein zweckgerichtetes, perlokutiven Zwecken unterworfenes Handeln voraussetzt, bei dem - oft neben weiteren Funktionen - eine zumindest minimale Informationsübertragung immer konstitutiv ist, besteht zwischen Kommunikation und Kognition keine Trennung, sondern setzt Kommunikation Kognition voraus. Kommunikatives Handeln mit Ziel Information wird erst dann glücken, wenn die Mitteilung als mittels semiotischer Zeichen, darunter vor allem sprachlicher, im Text/Diskurs materialisiert verfügbares Informationsangebot im weiteren Sinne, kognitiv adäquat durch den Rezipienten verarbeitet bzw. in seinem Gehirn konzeptuell koaktiviert wird. Bei kommunikativen Handlungen etwa mit inzitativ-direktiver Zwecksetzung, kommt hinzu, dass zusätzlich zum Mitteilungsgehalt auch die appellative Mitteilungsabsicht/ kommunikative Sinngebung des Senders, etwa auch unter Zuhilfenahme von Kontextwissen, entsprechend erkannt wird.

Kommunikation und Kognition bilden also eine letztlich untrennbare dialektische Einheit, die wesentliche Bedeutung für die Sicherung der Spezies Mensch, für das Überleben und die Anpassung der Menschen an sich ändernde Umweltbedingungen und die Gewährleistung der unverzichtbaren sozialen Interaktion besitzt.

1.3.5 So wenig wie Kommunikation und Kognition als Gegensatzpole verstanden werden können und darüber die Bedeutung von natürlichen wie auch von künstlichen 
Sprachen und sonstigen Zeichensystemen vergessen werden darf, so wenig ist zu übersehen, dass kulturelle Aspekte, die ja im weitesten Sinne gewisse Regularien für soziales Interaktionsverhalten als Konventionen/Normen, Handlungs-, Interpretationswie Verhaltensanleitungen für nonverbale, aber eben auch für verbale, kommunikative Interaktion vorgeben und Anlass zur sprachlich-semiotischen Erstnomination/Bezeichnungsgebung darstellen, einen bedeutenden Einfluss auf Kognition, Kommunikation und demzufolge auch auf Sprache ausüben, und zwar sowohl als System (hier besonders etwa hinsichtlich der Bereitstellung von Lexemen, deren Polysemie und Dichte/ Konkretheit sowie Eingebundenheit in größere semantisch-kognitive Zusammenhänge) als auch als Verwendung (habla, norma nach COSERIU 1988; hier etwa Textsortenkonventionen als Konstruktionsvorgaben/ Type für je konkrete Textvorkommen/ Token) ausüben.

1.3.6 Die aufgezeigte enge Verwobenheit von Kultur-Interaktion-KommunikationKognition und Sprache ${ }^{5}$ manifestierte sich u. a. in der in (3) gegebenen schematischen Darstellung und in den von uns kurz referierten bisherigen Überlegungen/Untersuchungen.

\footnotetext{
${ }^{5}$ Verstanden als materiell-kognitive Größe und ausgezeichnetes Charakteristikum menschlicher Kognition (vgl. BIERWISCH 1999).
} 
(3) Abbildung aus einem Vortrag von KALVERKÄMPER gehalten in Leipzig 2002:
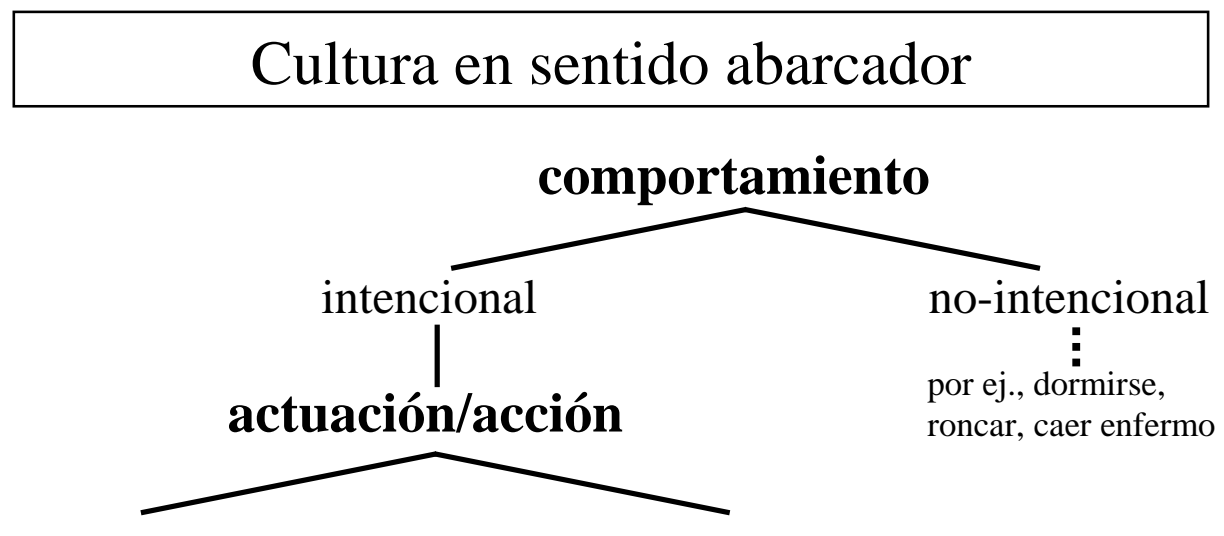

relacionada con otro participante

no relacionada con otro participante

p.ej. leer un libro...

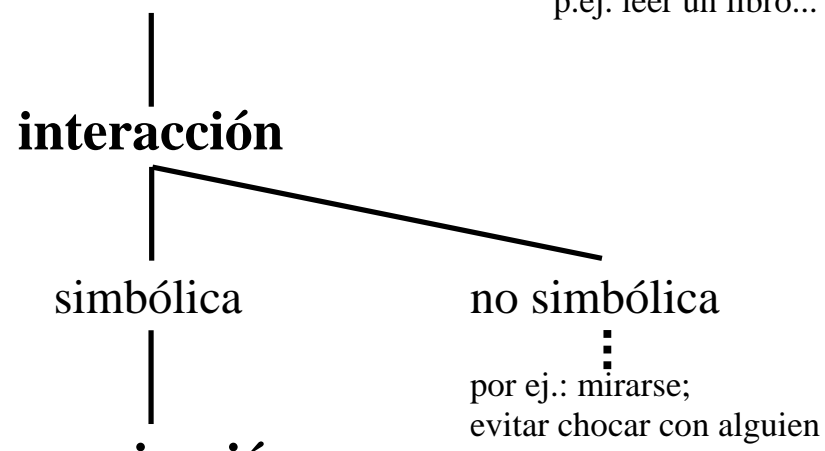

comunicación

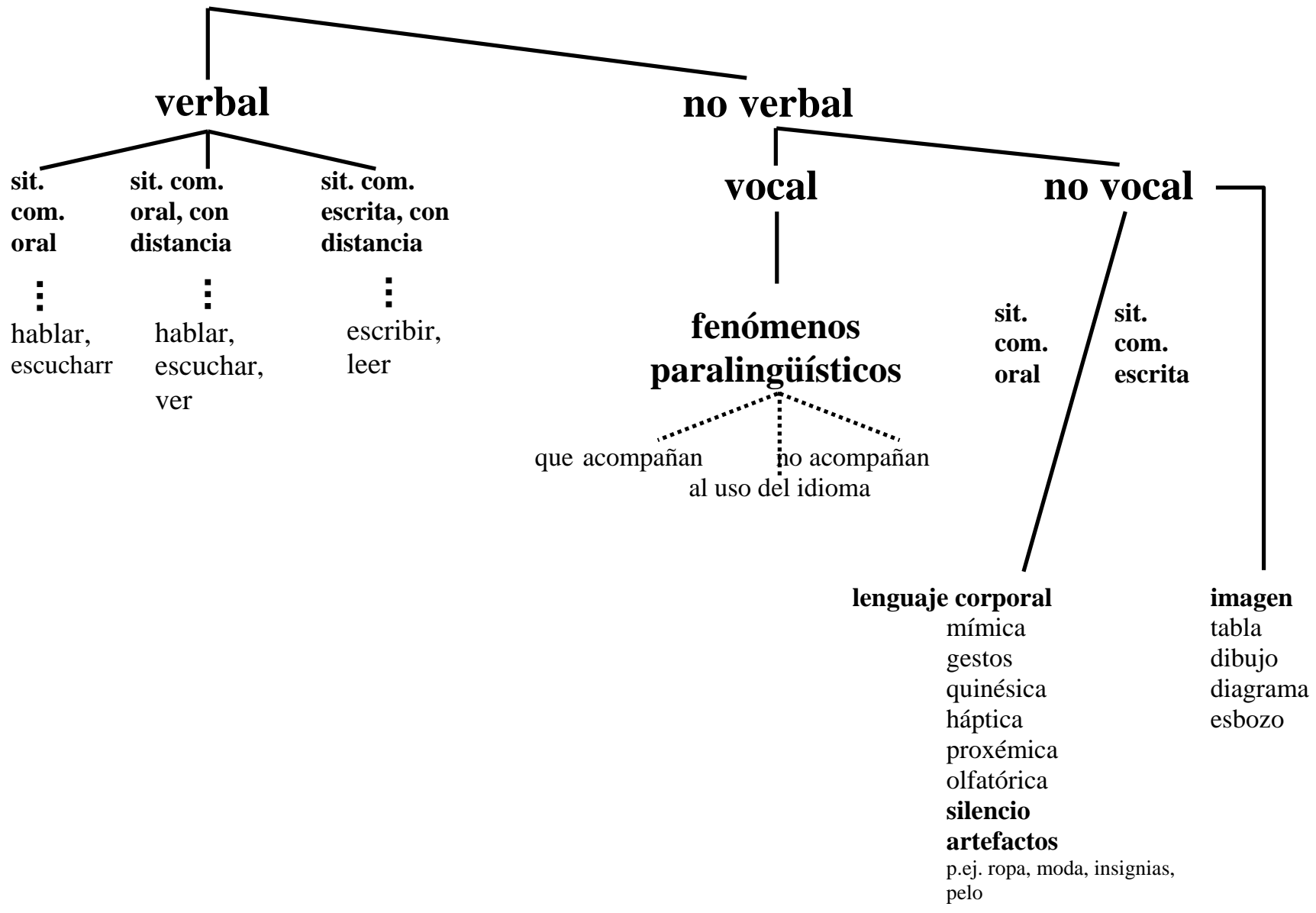


2. Angesichts der recht summarisch aufgezeigten Interrelationen zwischen kulturellen, kognitiven und kommunikativ-sprachlichen Phänomenen wird immerhin bereits deutlich, dass im weiteren Fortgang unserer Darlegungen wohl nur der Frage nachgegangen werden kann, in welcher Weise, anhand welcher Aspekte der komplexen translatorischen Aktivität sich kulturelle wie kognitive Aspekte besonders deutlich nachweisen lassen. Vielleicht sollte es sogar angesichts des Umstandes, dass Kognitives wie aber auch Kulturelles letztlich alle kommunikative wie translatorische wie soziale Interaktion in der einen oder anderen Weise prägen, Kognitives wie Kulturelles also gewissermaßen omnipräsent oder zumindest dominant präsent erscheinen, einfacher sein zu prüfen, ob es Restbereiche und wenn ja, welche, geben dürfte, wo deren Wirken nicht so offensichtlich nachgewiesen werden kann.

2.1 Natürlich hängen Aussagen zur Verwobenheit kognitiver und kultureller Faktoren im Translationsprozess und zu deren Manifestation in Translaten nicht unwesentlich davon ab, wie im Einzelnen Kulturelles und Kognitives bestimmt werden. Eine detaillierte Darlegung zu diesen durchaus kontrovers bzw. divergierend bestimmten Termini würde indes sicher zu weit abführen von dem hier selbst gestellten und möglicherweise doch etwas zu prätentiösen Ziel. Immerhin wird bspw. von ca. 400 Definitionen von Kultur gesprochen (dazu u. a. auch HAUSER 2007), wobei sich naturgemäß wohl durchaus gewisse übereinstimmende Bestimmungsmerkmale ausmachen lassen dürften. Auch hinsichtlich der Bestimmung von Kognition bestehen Divergenzen, die angesichts des Booms der kognitiven Wissenschaften und der kognitiven Wende in der Linguistik nicht verwundern; wir begegnen demzufolge auch hier Definitionen bzw. Vorstellungen von Kognition, die in Intension und Extension mehr oder minder voneinander ebenso abweichen wie methodische Zugänge und hypothetische Grundannahmen für Kognition bzw. auch für Kultur.

2.2 Wir können im Folgenden, ohne jeglichen Anspruch auf Vollständigkeit noch auf eine systematisch-hierarchische Darstellung, nur einige ausgewählte Aspekte zur angeschnittenen komplexen Problematik aufzeigen.

Dabei werden kulturelle Faktoren im Folgenden einen etwas breiteren Raum einnehmen als kognitive. 
2.2.1 Wird Kultur so allgemein verstanden wie in Abbildung 3 reproduziert, so stellt sich in der Tat die epistemologisch wie philosophisch-ontologisch relevante Frage, ob es wohl Teile der menschlichen Konzeptualisierungsleistungen, also der Weltsicht, der Weltwissensrepräsentationen, geben kann, die nicht kulturell geprägt sind. Da Kognition aufs engste mit Sprache verknüpft, ja Sprache ohne Kognitionsleistung ebenso wenig denkbar ist wie ohne ihre Leistung beim interpersonellen Wissenstransfer und bei der soziale wie diakulturelle Gruppen überschreitenden Kooperation, also in der Kommunikation innerhalb einer Parakultur, darf gemutmaßt werden, dass sich Kulturelles auch in Sprachlichem manifestiert.

Wir können an dieser Stelle uns allerdings nicht anheischig machen zu entscheiden, ob wirklich alle Spezifika sprachlicher Elemente wie Strukturen in letzter Instanz kulturbedingte Ursachen haben. Bislang hat die Forschung zu kulturellen Aspekten und deren Wirkung auf Kognition und Kommunikation, so sie sich nicht auf die Beschreibung solcher kultureller Aspekte überhaupt beschränkte, allerdings u. W. vor allem nach Divergenzen und weniger nach Gemeinsamkeiten zwischen den Kulturgemeinschaften und Kulturkreisen gesucht, wobei sie damit in einem gewissen Widerspruch etwa zur Universaliensuche hinsichtlich linguistischer wie kognitiver Sachverhalte steht. Man geht wohl auch nicht fehl, wenn man anmerkt, dass seitens der Linguistik wie aber auch dominierender Bereiche der Kognitionsforschung der mögliche Einfluss kultureller Faktoren auf die Weltsicht/ Kognition unzureichend thematisiert oder aber verkürzt/ einseitig verabsolutierend bspw. als Wirkung der Sprache, als sprachbedingt, betrachtet wurde. Angesichts der bedeutsamen Rolle, die die Frage nach einer sprach- und/oder kulturgeprägten Weltwissensrepräsentation (Weltsicht/Weltanschauung) mit tief verwurzelter Relevanz für die Kommunikation, insbesondere das Verstehen/ Verständlichmachen, d.h. die Evokation von Weltwissenskomplexionen, für die Begründung der Übersetzbarkeit bzw. deren Inabredestellen besitzt ${ }^{6}$, muss es verwundern, dass sich zu diesem Thema selbst aus kultureller Sicht, ganz besonders aber von translatologischer Warte aus, bislang kaum detailliertere neuere Überlegungen finden. In diesem Kontext könnte die kulturelle Wende in der translatologischen Forschung heilsame Impulse auch für die Lösung einer

\footnotetext{
${ }^{6}$ Vgl. dazu als prominenter Vertreter besonders Humboldt, s. aber auch WEISGERBER 1950 neben Sapir/Whorf u.a.m.
} 
so fundamentalen Problematik geisteswissenschaftlicher Forschung geben (vgl. insbesondere WITTE 2000, aber auch VIDAL 1995; 1998).

2.2.2 Bei der Behandlung kultureller Aspekte (dazu u. a. WоTJAK 1993a, b, 1984; WITTE 2000) gilt es in Rechnung zu stellen, dass es selbst im Rahmen ein und derselben Kultur- und Kommunikations- wie Sprachgemeinschaft bei intensiver sozialer Interaktion mehr oder minder deutliche Unterschiede gibt hinsichtlich zumindest von Teilbereichen kulturell geprägter Einstellungen zu Sachverhalten, deren Wertung und Wichtung wie aber auch deren sozialen Interaktionsverhaltens und der Akzeptanz sozialisierter, tradierter Konventionen, Sitten und Gebräuche. ${ }^{7}$

Betrachtet man die selbst innerhalb einer relativ monolithischen Personengruppe, die unter wesentlich gleichen sozioökonomischen, politischen, administrativen, klimatischgeografischen, kulturell-bildungsmäßigen Umfeldbedingungen und geteiltem Wissen um gemeinsame Traditionen, Sitten und Gebräuche, etc. in tagtäglicher Interaktion lebt, also den so genannten soziokulturellen Hintergrund (social cultural background) teilt, weiter bestehenden Divergenzen, so nimmt es nicht wunder, dass zwischen Sprach- wie Kommunikationsgemeinschaften, die in einem anderen soziokulturellen Umfeld leben und interagieren, sich ein mehr oder minder großes soziokulturelles Differential auftut. Mit diesem soziokulturellen Differential wird eine ganze Gruppe von Belegexemplaren für eine extensionale Begriffsbestimmung von Kultur benannt, die sich in divergierenden kulturellen Modellen, Schemata, Szenen- wie Szenarien, etc. niederschlagen, wobei durch Neubert dieser Begriff auch Eingang in soziolinguistische Betrachtungen gefunden hat (vgl. GROßE/NEUBERT 1974).

2.3 Dieses soziokulturelle Differential umfasst in einem sehr, möglicherweise auch zu weiten Verständnis faktisch alle vom Menschen vorgefundenen und gestalteten Lebensumfelder, dessen Alltagsleben und Interaktion in Beruf und Freizeit (dazu u. a. WotJAK 1993a, b; 1994). Deren Konzeptualisierung kann unter dem Einfluss übereinstimmender Weltauffassungen, Ideologien, religiös geprägter Moralgrundsätze und Wertesysteme sowie von Austauschkontakten über Jahrhunderte hinweg und

\footnotetext{
${ }^{7}$ Vgl. die Hinweise auf eine Idiokultur, auf Familiokulturen, Gruppenkulturen, aber bspw. auch auf Firmenkulturen (Diakulturen), etc., neben bzw. im Rahmen einer gemeinsamen Parakultur, wobei kulturelle Faktoren, etwa neben sprachlichen, in denen sie sich in mannigfaltiger Weise niederschlagen,
} 
besonders angesichts der neuen Tendenzen und Möglichkeiten zur Globalisierung u. U. aber auch beträchtliche Gemeinsamkeiten aufweisen, von ideologisch oder auch beruflich bedingten Übereinstimmungen in den Konzeptualisierungen der Welt (darunter neben propositionalem Wissen auch konnotativ-wertende Wissenstatbestände) zwischen bestimmten Gruppen Sprach- und Kulturgrenzen überschreitend ganz abgesehen.

So wie man zu Recht u. a. auf die Gruppen integrierende, also nicht nur signalisierende Funktion von Sprache (bspw. Jugendsprache) hingewiesen hat, gilt es zu beachten, dass auch kulturelle Aspekte sehr wesentlich sind für Integration und Identitätsgefühle und dass nach vielfältiger Überzeugung bspw. sprachliche Fehlleistungen bei weitem nicht so störend empfunden werden wie etwa Verstöße gegen zumeist unbewusst geteilte, normierende kulturelle Gepflogenheiten/ stereotyp befolgte Verhaltensmuster, Archetypen des Verhaltens wie auch von dessen Bewertung. So ist es bspw. im Deutschen eher üblich, dass sich Angerufene mit Namen identifizieren, eventuell zunächst mit Institution/Firma + Namen; im Spanischen ähnlich dem Französischen jedoch allgemein ohne Namensnennung des Angerufenen im ersten Gesprächs-Turn.

2.3.1 Ebenfalls einzuordnen in die soziokulturelle Teilsachverhaltsdomäne "soziale Interaktion” wären solche üblichen, prototypischen Interaktionssituationen, wie bspw. die Anrede (mit starker sprachspezifischer wie intralingualer Ausdifferenzierung - vgl. sich daraus ergebende Übersetzungsprobleme unter (4)), bei Begrüßungsritualen, d.h. nonverbal-verbalen Behavioremen/ Kulturemen, die sich u. a. im kulturgeprägt abweichenden Verhalten beim Geben von 1-3 Küssen zwischen französischen und spanischsprachigen Sprechern manifestieren (mit welcher Wange wird begonnen?) oder aber auch in nachstehenden Beispielen, bei denen abweichende Höflichkeitskonventionen als wichtige Bestandteile der mitunter vernachlässigten phatischen Funktion kommunikativer Interaktion (dazu NoRD 2002b) zum Tragen kommen:

(4) Vgl. etwa Monsieur, vous avez perdu vos gants; Doña/Don, SeñoritaFräulein/Frau; compañero; in deutschen Übersetzungen bspw. M. de Savigny, Herr de Savigny vs. Herr von Savigny; Meister, Sportsfreund; mein Gutester, etc.

eingefroren, finden, einen bedeutenden Beitrag zur Integration wie Identitätssicherung der interagierenden erkennenden wie kommunizierenden Subjekte leisten. 
Analog wird interkulturelles Wissen aktiviert, etwa bei dem hier nicht näher auszumalenden, landeskundlich sehr instruktiven Beispiel abweichender Behavioreme/ Kultureme Spanisch/Deutsch beim Empfang/der Überreichung von Geschenken (dazu u. a. OKSAAR 1988; GERZYMISCH-ARBOGAST/MudERSBACH 1997; WitTE 2000).

2.3.2. Weitere Teilbereiche aus dem komplexen Lebensumfeld, aus dem eine soziale Interaktion in der Regel kaum ernsthaft wegzudenken ist, wären z.B. geografischklimatische Phänomene ${ }^{8}$, kulturgeprägte Präferenzen hinsichtlich von Lieblingssportarten/ Freizeitbeschäftigungen ${ }^{9}$, Speisen und Getränke, also der Bereich der Alimentation/Gastronomie, aber auch religiös geprägter Unternehmungen und Institutionen. Vgl. dazu nachstehende völlig unzureichende Liste sogenannter Realienlexeme, d.h. von Bezeichnungen für typische, “endemische”, also landes- bzw. sogar regionenspezifische Phänomene, denen in einer anderen Kultur- wie Sprachgemeinschaft zumeist kein Konzept bzw. keine bereits sozialisierte Bezeichnung entspricht, so unter (5).

(5) Corrida; romería, Semana Santa; gazpacho, sangría; queso manchego, jamón serrano; paella; ESBEC; cederista (Kuba); Mistral; Cognac, Champagner; Leipziger Allerlei; Plauener Spitzen/ Aachener Spitzen; Labskas; Doppelspänner (Kaffee); Broiler, Rotkäppchen (beliebte Sektmarke Ost) ...

Dass es sich dabei um letztlich kulturgeprägte Phänomene handelt, zeigt, dass bspw. auch Realien innerhalb einer Sprachgemeinschaft auftreten ${ }^{10}$; so ist bspw. die in Kuba (aber auch Argentinien) so übliche boda de los quince oder auch deren verkürzte Bezeichnung als los quince auf der iberischen Halbinsel faktisch unbekannt. In hohem Maße länderkulturspezifisch erscheinen aber auch Bildungsinstitutionen und abschlüsse, was bspw. die Übersetzung solcher Dokumente wie Zeugnisse, Lebensläufe, etc. in zusätzlichem Maße kompliziert bzw. deren länderübergreifende Verwendung im gleichen sprachlichen Umfeld erst durch verstehensfördernde Kommentare/Vergleiche mit den in der Zielgemeinschaft üblichen Bildungsabschlüssen wirklich möglich macht.

\footnotetext{
${ }^{8}$ Vgl. dazu u. a. Fleischmann 2001 unter Bezug auf russisch-deutsche Übersetzungen.

${ }^{9}$ Vgl. das exotisch anmutende Kricket der Engländer, der Stierkampf in Spanien; Hahnekämpfe in Lateinamerika, etc.
} 
2.3.3 Stark kulturell bzw. genauer politisch-ideologisch bzw. auch moralisch-ethisch geprägte Phänomene zählen in einem weiten Verständnis ebenfalls zum soziokulturellen Hintergrund, wobei auch hier das zum Verstehen erforderliche einschlägige Wissen in der Zieltextgemeinschaft in der Regel nicht vorhanden ist oder aber zumindest nicht in toto, bspw. hinsichtlich aller von einer bestimmten Kulturgemeinschaft mitassoziierten Konnotationen /Bewertungen koaktiviert wird, wären bspw. solche unter (6) aufgeführte Bezeichnungen für zumindest teilweise inzwischen von der gesellschaftlich-politischen Entwicklung überholte Sachverhalte zu erwähnen. Was diese Bezeichnungen (beinahe schon als Historismen zu bezeichnen) anbetrifft, so dürften sich selbst innerhalb des Deutschen bestimmte Wissensdefizite nachweisen lassen, die von Unkenntnis bis zu einer nur sehr annähernden Kenntnis und/oder abweichenden Bewertung reichen, von Verständnisproblemen beim Wechsel ins Spanische ganz abgesehen (vgl. Übersetzung von Grass“ Ein weites Feld“).

(6) a) Begrüßungsgeld; Wendehals; Seilschaften; aufrechter Gang; Mauerspecht; Aluchips; Treuhand; Normannenstraße/Runde Ecke; GST; MTS; HO; MfS; NVA; NAW; DHFK, etc.

b) Jugendweihe, Kindertagesstätte/Kita; Kaufhalle, Broiler; drüben und hüben; Ossi, Wessi; Ostalgie (Nostalgie nach der DDR), etc.

c) Peanuts; ein weites Feld; die Republik jähnt, etc.

In den angesprochenen Teilbereich soziokultureller Phänomene fallen u. E. aber auch die so genannte political correctness als Leitlinie der Interaktion zwischen Menschengruppen, insbesondere auch zwischen unterschiedlichen Staaten, Tendenzen zur Verhüllung bzw. Verschleierung von Sachverhalten durch vage bzw. das Sozialprestige der Sprachbenutzer schonende (face saving-Aspekte) Bezeichnungen sowie solche zur Vermeidung von negativen Wertungen oder aber Sprachregelungen im Dienste diplomatischer Nichtanerkennung von bestimmten politischen Realitäten, wie u. a. solche unter (7) angeführte Bezeichnungen ${ }^{11}$.

(7) Vgl. in lateinamerikanischen Dokumenten die "verhüllende" Bezeichnung cooperación económica; in Dokumenten der EU dafür Bezeichnungen wie aid oder assistance; vgl. auch die Ersetzung von Roter Armee durch Sowjetarmee

\footnotetext{
${ }^{10}$ Vgl. Beispiele unter (3), aber auch abweichende Textsortenkonventionen zwischen Kuba und Spanien, also innerhalb der spanischsprachigen Länder.

${ }^{11}$ Vgl. hierzu auch Schriften von Stuart CHASE und Georg KLAUs 1964 zur Macht des Wortes und viele Untersuchungen zur kontrastiven Pragmatik.
} 
(Vermeidung der dominant negativ bewerteten Farbbezeichnung "rot"); aber auch Sowjetische Besatzungszone/SBZ, Pankower Regime, etc.

Wahrscheinlich unter dem Einfluss spezifischer Erziehungsideale (educación formal, gute Manieren, angemessenes Sozialverhalten, etc.) haben sich bestimmte Verhaltensweisen herausgebildet, deren Unkenntnis als Erklärungshintergrund zu Missverständnissen führen kann, die u. U. schon vorhandene archetypisch-stereotype Vorbehalte/ Vorurteile nähren bzw. aus diesen interpretatorisch gestützt werden. So bspw. bei Abweichungen im Falle des dem Sender offensichtlich in besonderem Maße peinlichen Ablehnens des Vorschlages eines Gesprächspartners, also letztlich bei abweichenden Höflichkeitskonventionen. Bekannt ist bspw., dass man in Thailand/Laos einem Kind nicht über die Haare streichen soll, dass in anderen Kulturkreisen die Fußsohlen nicht auf den Gesprächspartner zeigen dürfen, man seiner Schwiegermutter den Rücken beim Sprechen zukehren, man einem Japaner nicht in die Augen sehen sollte, etc. Weniger deutliche, aus Erfahrung erworbene (kritische) Einschätzungen von Verhaltenspräferenzen wären bspw., dass sich Deutsche im Verständnis von Schweden zu oft direkt in Dinge einmischen, die sie nach deren Überzeugung nichts angehen (STEDJE 1988 - nicht bei Rot über die Straße gehen als Ermahnung); zu laut sprechen, dass Finnen im Allgemeinen wenig sprechen und längere Gesprächspausen nicht unbedingt ein Zeichen von fehlendem Interesse am Gespräch sind. In diesem Zusammenhang wird noch ein zusätzlicher, offenbar eng mit den kulturellen Aspekten verflochtener Problemkreis im Umfeld von Mentalitätsstudien, Forschungen zur Völkerpsychologie ${ }^{12}$ angerissen, dessen Erörterung uns hier jedoch zu weit ab- und zudem auf ein problematisches, bisher kaum detaillierter und mit objektivierten bzw. objektivierbaren Methoden beschriebenes Nebengleis mit Relevanz für den Sprachvergleich, die kontrastive Pragmatik, Rhetorik und Stilistik wie aber letztlich auch die Translation, führen würde.

Nicht sofort einsichtig und wohl aus unserer eigenen Kultur heraus, der wir letztlich kaum jemals wirklich völlig entraten können, kaum akzeptabel erscheint es hingegen, wenn bspw. in Lateinamerika (so eine persönliche Erfahrung) ein einfacher Straßenkehrer sich scheute, seine Unwissenheit hinsichtlich einer Adressenanfrage zu

\footnotetext{
${ }^{12}$ Vgl. etwa die Analysen Wundts; diese wären u. E. mit Augenmaß neu aufzunehmen, wobei bspw. auch solche offenbar oft zu kurzschlüssig generalisierenden Aussagen von Malblanc 1968 in seiner vergleichenden Stilistik, aber auch von Lerch 1933 zum französischen Volkscharakter als "kühle Rechner”, die zu keinen tieferem Empfinden fähig seien, daher fehlte dort eine lexikalische Entsprechung für Gemüt, Gemütlichkeit, etc.
} 
bekunden und den Fragenden im Brustton der Überzeugung in eine gemutmaßte, aber falsche Richtung schickte. Komplex sind aber auch die bei Äußerungen bei (8) ablaufenden Inferenzen auf das leider abweichende kulturelle Hintergrundwissen/ Höflichkeitsverhaltensmusterwissen ${ }^{13}$. Dies führt dazu, dass der deutsche Muttersprachler sich schwer tut, die dort getroffene Aussage, im kubanischen Spanisch noch witzig pointiert aus dem speziellen Wissen, als das zu interpretieren, was sie im konkreten Äußerungskontext sein sollten, nämlich eine höfliche, face-saving gewährleistende, aber nicht weniger definitive Ablehnung. Mit anderen Worten, das mit den angeführten Sätzen - zudem weitgehend kontextunabhängig - zum Ausdruck Gebrachte entspräche vielmehr einer verbalen Formulierung: no lo pensará nunca más, d.h. dass nicht mehr darüber nachgedacht wird. Man kann sich die aus dem Nichtverstehen dieser kommunikativ-illokutiven Funktion der spanischen Äußerungen ergebenden Weiterungen vorstellen: der Deutsche wird einige Zeit zuwarten, nicht zuletzt weil er akzeptiert, dass man auf Entscheidungen länger warten muss. Er wird aus Interesse und ohne schlechtes Gewissen nach einiger Zeit unbefangen nachfragen, ob der spanische Partner es sich überlegt hat. Letzterer wiederum wird dies als einen schockierenden Vorgang empfinden ${ }^{14}$ und die Nachfrage auf das Konto fehlenden Taktgefühls schieben. Der Deutsche wird bei eintreffender, nunmehr eindeutiger Rückantwort sich u. U. in seinen Vorbehalten bestätigt sehen, dass man den Worten spanischer Partner sehr skeptisch, ja misstrauisch gegenüber stehen sollte. Analog muss die Äußerung brasilianischer Gesprächspartner nicht wörtlich als Zusage, sondern vielmehr als Ankündigung, dass keine Reaktion erfolgen wird, verstanden werden.

Voy a pensarlo. Ya (me) lo pensaré. Lo pasaremos al organismo superior (Kuba). Te ligarei amanhã.

2.3.4 Z.T. bereits gestreift (Wendezeit wie Vorwendezeitsphänomene ohne Kontinuität bis ins Heute) wurden historische Sachverhalte, deren Wissenserwerb, -ausprägung und -auswahl zudem zumeist in spezifischer Weise kulturell-ideologisch-nationalistisch geprägt erscheinen. Dass dabei an einem gleichen Sachverhalt unterschiedliche Aspekte akzentuiert und abweichende Bewertungen vorgenommen werden, und dies nicht etwa

\footnotetext{
${ }^{13}$ Spanier neigen tendenziell stärker zu einer indirekten Mitteilung einer Ablehnung, vergleichbar etwa den Japanern, für die eine Ablehnungshandlung ganz unangebracht erscheint.
} 
sprach- sondern kulturgeleitet, liegt so offensichtlich auf der Hand, dass es wohl keiner näheren Illustration bedarf. Die abweichende Entwicklung im vereinten Deutschland führt dabei zu immer wieder verblüffenden, weil z. T. gar nicht erwarteten Divergenzen/Nichtkongruenzen, auch bei der Einschätzung von historischen Phänomenen und selbst fortwirkend bei der Bewertung aktueller Ereignisse. Dabei fällt im Übrigen auch die abweichende Sozialisierung schon ab dem Kindesalter als in der interkulturellen Kommunikation der Nachwendezeit bis heute in Rechnung zu stellende Größe ins Gewicht, da sie als Erklärungshintergrund für Mentalitätsunterschiede, abweichende Verhaltensweisen und Befindlichkeiten, Präferenzen bei der Bewertung von Verhalten, etc. führt. Hierhin fallen bspw. auch Nichtkongruenzen hinsichtlich des Wissens an kulturellen Phänomenen im engeren Sinne: so abweichende Lektüre in Schule und Freizeit, abweichende Musik- und Kunsterlebnisse sowie als Vorbild ausgegebene Kunstrichtungen ${ }^{15}$.

2.3.5 Wie die sicher noch unvollständige Aufzählung verdeutlicht, werden hier existenziell sehr bedeutsame, prägende Aspekte für eine bestimmte Kommunikations-, Sprach- wie Kulturgemeinschaft angesprochen, deren Kenntnis gerade angesichts des hohen Grades an Unbewusstheit und Verwurzeltheit in den erkennenden und interagierenden Subjekten und der unbeabsichtigten, damit aber nicht geringeren, störenden und zumeist unbewusste emotionale Akzeptanzvorbehalte hervorrufenden Gegenreaktion selbst gegenüber rational einleuchtenden Argumentationen, bei der verstärkt statthabenden interkulturellen Kommunikation von zunehmend anerkannter Relevanz ist. Dabei schlägt sich der soziokulturelle Hintergrund nicht nur nieder in den sprachlich indizierten konkreten Prozessen, Erscheinungen ausgewählter Teilbereiche, also in den Konzeptualisierungen, den lexikalischen Bedeutungen und hier etwa im propositional-denotativen Bereich, sondern bspw. auch im konnotativ-bewertenden, emotive Aspekte koaktivierenden Bereich der Bedeutungen solcher Kulturindikatoren/ Realienlexeme oder auch weiterer LE. Der Einfluss kultureller Faktoren auf kognitivsemantische Phänomene manifestiert sich darüber hinaus aber bspw. auch in der bevorzugten Konzeptualisierung ausgewählter Bestimmungsmerkmale, in

\footnotetext{
${ }^{14}$ Immerhin hatte er doch nach seinem Empfinden hinreichend verdeutlicht, dass er nicht mehr darüber nachdenken wollte, die Angelegenheit also abschlägig beschieden sei.

15 Bspw. die so genannte entartete Kunst im Nationalsozialismus, der sozialistische Realismus und Vorbehalte/Unverständnis gegenüber der abstrakten Malerei in der DDR, etc.
} 
abweichenden Zuordnungen von LE bzw. den durch diese bezeichneten Sachverhalten zu Klassenbildungen, in abweichender Auswahl prototypischer Vertreter für Klassenbildungen (bspw. Werkzeuge/Hämmer - vgl. weitere Beispiele unter 9), in dem Interesse an einer spezifizierten, d.h. die Phänomene der bezeichneten Umwelt stärker untergliedernden Bezeichnungsfülle (bspw. höhere oder geringere Dichte von lexikalischen Belegen für begrifflich-semantische Felder - vgl. Beispiele unter (10)).

(9) a) Vgl. u.a. die Abweichungen hinsichtlich der als Prototyp gewählten Exemplare von Hammer in den USA und Deutschland; vgl. aber auch die denotativ-konnotativen Divergenzen bei solchen Alltagsbezeichnungen wie pain/pan (Weissbrot in Baguetteform; faktisch zu jedem Essen gereicht) - dt. Brot; vin (vgl. Rituale bei Auswahl im Restaurant), vino und Wein; fromage - Käse (unter der Berücksichtigung, dass fromage jedes warme Essen abschließt); café \# Wiener Kaffeehaus (eher Gasthaus); control sowie dt. Kontrolle...

b) vgl. die Übersicht über abweichende Zuordnungen von Autos zu den im Prinzip übereinstimmenden Klassen in Deutschland und den USA (besonders Mittelklasse/MID-SIZE CARS) in SCHMITT (1999: 273); vgl. aber auch signifikante Abweichungen bei Angaben zum Kraftstoffverbrauch: in Deutschland Liter $/ \mathrm{km}$, in den USA zurückgelegte Wegstrecke in Meilen (mileage), wobei factors that contribute to lower mileage are... im Deutschen wiedergegeben werden müssten als: Faktoren, die zu einem höheren Verbrauch führen, sind ...(SCHMITT 1999: 277).

(10) Vgl. das Feld der Fortbewegungsverben im Deutschen gegenüber dem spanischen oder auch französischen Feld sowie die generelle Tendenz des Französischen/ Spanischen zur Verwendung abstrakterer Bezeichnungen generell - Beispiele enjeu, action; agent; aménagment; conditionnement; contrainte; engagement; expansion; génie; gestion; opération; promotion; recrutement; surenchère, etc., aber bspw. auch fårmor/mårmor (schwedisch) $=$ Großmutter väterlicherseits/ mütterlicherseits bzw. Bezeichnungen für älteren bzw. jüngeren Bruder (aber nicht für Bruder an sich) im Ungarischen, die mehr als 200 Bezeichnungen für Pferderassen/ und -farben bei den Gauchos (und französischen Pferdezüchtern) sowie das viel zitierte Beispiel mehrerer Bezeichungen für <Fortbewegung auf einem "spezifisch beschaffenen" Schnee> in den Eskimosprachen (dazu schon detailliert u. a. schon MounIN 1963).

2.4 Zusammenfassend kommt (Sozio)Kulturelles kognitiv wie sprachlichkommunikativ in der situativ-diskursiven Äußerung einer senderintendierten wie rezipientenrealisierten Botschaft ${ }^{16}$ vor allem in nachstehender Weise zum Ausdruck:

\footnotetext{
16 Vgl. zur Interrelation von Senderintention/Meinung/ Sinngebung und Empfängerdeutung/ Sinnerfüllung Details bei WOTJAK 1985; 2003; vgl. Details zum kommunikativen Sinn als Richtwert für die Translation und zur Interrelation von Mitteilung/Botschaft als komplexer Interaktion von Gesagtem/Vertextetem (posé) und Mitverstandenem (supposé) sowie komplementär überlagerndem Gemeintem in WOTJAK 2005b.
} 
2.4.1 In Form sememisierten, d.h. anteilig in die lexikalische Bedeutung eingefrorenen, sozialisierten und usualisierten Weltwissens, hier eines Einzelsachverhaltswissens O’kult. Dabei wirken kulturelle Aspekte darauf ein, welche Merkmale/Eigenschaften als konzeptuell wie kommunikativ relevant selektiert werden, welche Tiefenschärfe bei der Bezeichnung/ Sachverhaltswissensrepräsentation wie Bedeutungskonstitution als erkenntnis- wie bezeichnungsrelevant angesehen und demzufolge mit einer bestimmten generischen oder auch vagen LE bzw. auch mit mehreren konkreteren Bezeichnungen als Semantik verknüpft wurde. Bei diesem direkten, durch die lexikalischen Einheiten/LE qua Bedeutung indizierten $\mathrm{O}^{\prime}$ kult-Konzeptualisierungen gehen über kommunikative Austauschbeziehungen eine mehr oder minder geteilte Anzahl und Auswahl propositional-denotativer Wissenskomponenten in den Bedeutungskern ein. Daneben finden aber auch geteilte Bewertungen/ Einstellungen/ Emotionen als vergesellschaftete periphere Komponenten konnotativ-wertend-emotiver Art in den Bedeutungen ihren Platz, ja können diese sogar in toto selbst die Bedeutung konstituieren. In beiden Fällen fungieren die betreffenden LE dank ihrer Bedeutungen als direkte Indikatoren kultureller Phänomene, sei es nun für O’kult-Phänomene (kulturgeprägte Realien/Denotate) oder aber kulturell gebrochene Bewertungen von Phänomenen der Designatswissensdomäne O’soz in einem weiteren Sinne.

2.4.2 LE fungieren darüber hinaus aber auch als indirekte Indikatoren von soziokulturellem Hintergrundswissen insofern, als über deren Bedeutungen ohne direkten oder vermittelt-gebrochenen O’kult-Bezug kognitive Sachverhaltswissenskomplexionen koaktiviert werden als geteilter generischer Weltwissens- und/oder spezieller Situationswissensbesitz. Die dabei koaktivierten kognitiven Konfigurationen liefern als mehr oder minder komplexe Szenarien, kulturelle Modelle (Morillas/SÁNCHEz 1999), Schemata, Idealized Cognitive Models/ICM, Szenen, Folk Models, etc. über die LE (oft Namen von Persönlichkeiten des öffentlichen Lebens) nicht direkt koaktivierte, kulturell wie kommunikativ-kognitiv bedeutsame Hintergrundsinformationen (backgrounding) vor allem bei Anspielungen, also im Hinblick auf das neben dem Gesagten (posé nach DuCROT 1972) stets auch für die Kommunikations-/Kulturgemeinschaft kopräsente geteilte Mitverstandene (supposé nach DUCROT 1972 - dazu WOTJAK 2005b). 
Wie stark solches Szenen-, Skript- wie Szenarienwissen (Kulturem-, Holon- wie Holeme-Kenntnis nach OKSAAR 1988, GERZYMisch-ARBOGAST/MudERSBACH 1997) von landeskundlich-kulturellen Faktoren geprägt ist, wird etwa bei der Aktualisierung der Wissenskonfiguration deutlich, die bei der kognitiven Realisierung des Restaurantbesuchszenariums in Kuba, Spanien und Deutschland (besonders mit Bezug auf die ehemalige DDR) in kompetenten Sprechern koaktiviert wird. So wurde man bspw. in der DDR platziert, hatte also auch bei leerem Restaurant auf diesen Platzierungsakt des Obers zu warten; in Kuba rief ein Oberkellner - als capitán bezeichnet - nach Registrierung anhand einer Abfolgeliste die geduldig vor der Tür wartenden Gäste auf; andererseits unterscheiden sich die Bezahlungsmodalitäten nach wie vor deutlich zwischen Spaniern und Deutschen, etc.

2.5 Neben diesen letztlich über vorhandene LE mehr oder minder stark vermittelt oder direkt hergestellten Bezügen zu außersprachlichen soziokulturellen Sachverhalten, wirkt Kulturelles aber auch noch in vielfältig anderer Weise mit Kognition, Kommunikation und Sprache zusammen bzw. lässt es sich in sprachlichen Erscheinungen nachweisen. So dürften bspw. politisch-ideologische Gründe für den bevorzugten Gebrauch bzw. die Entstehung neuer Metaphern durch Übertragung von Bezeichnungen aus einer bestimmten Spenderdomäne (bspw. dem militärischen Bereich) bei Beispielen unter (11) als dominant betrachtet werden.

(11) a) Kampf für den Frieden/ die Erfüllung der Hauptaufgabe; die Dissertation verteidigen, Ernteschlacht; der General Winter; Waffenbrüderschaft; die Vorhut des Proletariats (DDR); aber auch jmdn. mit seinen eigenen Waffen schlagen, etc.; defender una tesis; Préstame una bala que estoy sin armamiento (Kuba - scherzhafter Kommentar beim Schnorren einer Zigarette);...

b) vgl. aber auch gewisse Präferenzen für erotische Metaphorik in französischen technischen Bezeichnungen wie:

$\begin{array}{ll}\text { Französisch } & \text { Deutsch } \\ \text { prise mâle } & \text { Stecker (elektr. Schnur) } \\ \text { prise femelle } & \text { Steckdose } \\ \text { bouchon femelle } & \text { Muffendeckel (Rohrverschluss) } \\ \text { raccord à bouts femelle } & \text { Innengewindekupplung }\end{array}$

c)Übernahmen medizinischen Vokabulars zur Bezeichnung ökonomischer Sachverhalte (Beispiele von SPILlNER 1994): la cancérisation de l'économie; ponctionner les revenus; les autorités monnétaires ont asphyxié les affaires; On appelle ça des points de fixation, des abcès. Le pus s'en écoule, et le corps est guéri. Le secteur manufacturier affiche une santé de fer, etc. 
Andererseits dürfte bspw. die in der Alltagssprache verallgemeinerte Verwendung von LE aus der Landwirtschaft bzw. bei Inselvölkern aus dem maritimen Bereich ebenfalls als letztlich kulturbedingt/-geprägt bezeichnet werden. Als solche könnte aber auch der bevorzugte Gebrauch bestimmter morphosyntaktischer Strukturen ${ }^{17}$; des Weiteren von so genannten personalisierten Konstruktionen ${ }^{18}$, von Kommentaren als expliziten Gliederungssignalen; von Explikationen (Gesagtem) und Implikationen (Mitverstandenem) bspw. in ausgewählten (populärwissenschaftlichen) Fachtextsorten im Englischen und Spanischen, gewertet werden.

2.5.1 In diesen Fällen geht es im Unterschied zu den unter 2.4.2 erwähnten faktisch nicht mehr um den Nachweis kulturellen Einflusses auf Sprache als SystemhaftLexikalisch-Fixiertes, sondern um Präferenzen im Gebrauch systemhaft vorhandener sprachlicher Mittel, also auf die Selektion wie Kombinatorik bereits vorhandener lexikalischer, morphosyntaktischer wie sonstiger sprachlicher Mittel bei der Konstituierung von Äußerungen/Diskursen/ Texten als Produkte der Parole. Dass hierbei insbesondere Textsortenkonventionen fürs Übersetzen relevant sind, liegt auf der Hand. Andererseits geht es aber auch darum, kulturgeprägte Unterschiede hinsichtlich der Bevorzugung bspw. indirekter Sprechakte zur Verwirklichung von Aufforderungshandlungen (12) herauszustellen, also Vorgaben für eine Kommunikationssituation wie Textsorten angemessene Präferenz ausgewählter Sprechakte/ Kommunikationshandlungen und -strategien zu ermitteln und diese bei der kommunikativ-situativen angemessenen ZS-Textproduktion zu berücksichtigen.

(12) a) indirekte Aufforderungssprechakte:

Steigen Sie auch aus? Ist das Fenster auf? (wenn dieses offensichtlich geschlossen oder geöffnet ist) Frau Müller, ich glaube, morgen müsste der Bericht abgesandt werden. Ein zweiter Versuch würde sich lohnen/schiene angebracht. ¿Porqué no me ayudas?

b) Lichtgeschützt aufbewahren. - Protéjase de la luz. Vor Gebrauch schütteln. - Agítese antes de usar. A la cama! A trabajar! - Ins Bett! An die Arbeit! Man nehme zwei Löffel Zucker, etc.

\footnotetext{
${ }^{17}$ bspw. statt des deutschen Vorgangspassiv die pasiva refleja oder weitere Passiversatzkonstruktionen im Spanischen, mit zunehmender Tendenz auch im Französischen.

${ }^{18}$ Dazu besonders FrIEDERICH 1969 mit Bezug aufs Englische und Gültigkeit auch fürs Französische.
} 
Schließlich zeigt sich der Einfluss von Kultur auch bei neuen Bezeichnungsbildungen und nicht zuletzt beim Verhalten gegenüber Bezeichnungsübernahmen, also - adaptierte oder nicht adaptierte - Entlehnungen (estándar bzw. standard als prestigeträchtigere Variante) bzw. deren Vermeidung durch Lehnübersetzungen, letztere etwa aus der kulturbasiert motivierten Angst vor Überfremdung des Französischen durch das Englische ${ }^{19}$.

2.5.2 Die kontrastive Rhetorik bzw. Stilistik / Pragmatik als bislang insgesamt noch relativ wenig entwickelte Teilbereiche der Linguistik sollten für den Translator und den Fremdsprachenunterricht/-lerner in besonderem Maße nützliche Informationen über Divergenzen bereit stellen können, die nicht nur Abweichungen hinsichtlich der Makrostruktur von Paralleltexten und etwa auch der Anordnung von Informationsblöcken, sondern auch auf Mikrostrukturen (bspw. Passiversatzkonstruktionen, bevorzugten "style verbal”; Autorenpräsentation / -ausblendung, etc.) sowie selbst hinsichtlich formal-typographischer Aspekte, so bspw. der Präferenzen hinsichtlich der Verwendung von Zeichnungen, etc. verdeutlichen.

So wird bspw. nach SCHMITT 1999 sprach- wie kulturunabhängig (weil die Corporate Identity als Firmenkultur beibehalten werden soll) in Werkstatthandbüchern von Mitsubishi eine große Anzahl bildhafter Hinweise anstelle der bspw. im Deutschen üblichen verbalen Indikatoren verwendet, was durchaus nicht der prototypischen Textsortencharakteristik im Deutschen gerecht wird und von den Werkstattmechanikern als störend, ja schockierend empfunden wird.

2.5.3 Kulturelles wirkt allerdings nicht nur hinsichtlich der präferierten Wahl bestimmter Sprechakte und möglicherweise auch hinsichtlich der Komponenten der Glückensbedingungen für solche Sprechakte; es wirkt vielmehr wohl auch in dem Sinne auf die Gestaltung einschlägiger Textsorten, als bspw. die Amerikaner/Engländer eine so genannte reader-oriented Darstellung bevorzugen, bei der der Sender dem potentiellen Leser mögliche Inferenzen auf dessen Weltwissen im Vorfeld weitgehend ersparen möchte, also etwa sonst zu erschließendes Vorwissen des supposé-Bereiches

19 Vgl. dagegen die davon deutlich abweichende, sprachkulturpolitisch von der Sprechermehrheit offenbar so sanktionierte Entscheidung für die Übernahme selbst von Luxusentlehnungen aus dem Englischen im Deutschen - dazu WOTJAK 2006b. 
dem Empfänger im wohlgemeinten Vorgriff schon als Gesagtes/posé gewissermaßen mundgerecht liefert. Gerade ein solcher leserfreundlicher und einfacher Stil ohne verschachtelte Sätze, etwaige Exkurse und sonstige Abweichungen von einem stringent durchsichtigen Aufbau, wird allerdings bspw. von spanischen Rezipienten solcher (populär)wissenschaftlicher Textsortenexemplare als allzu stark vereinfachend, den Leser ob der Simplizität der Darlegung unterschätzend, ja beleidigend angesehen. Ähnliches dürfte grosso modo zumindest auch für geisteswissenschaftliche Texte gelten. Inwieweit sich solche durch FERnÁndez POLO (1999), MonTAÑo-HARMón (1991) nachgewiesene Unterschiede in den Rezeptionsgewohnheiten durch konkrete Textanalysen als Nachweis von durch GALTUNG (1983), HiNDS (1987) behaupteten unterschiedlichen Wissenschaftsstilen stützen lassen, soll hier als weiterführendes Forschungsdesiderat nur angedeutet werden. Damit ist in keinem Fall auch etwas über die Zweckmäßigkeit bspw. eines so genannten teutonischen Stils gesagt, den ich in unrühmlicher Traditionsverbundenheit hier vorgeführt habe und der sich bspw. durch mangelnde sprachliche Eleganz/ Brillanz vom Gallischen unterscheidet.

3. Unsere bisherigen Darlegungen mit Akzentuierung der kulturellen Aspekte, darunter besonders des soziokulturellen Hintergrundes/ O’kult, dürften bereits die von uns postulierte enge Verwobenheit und große Bedeutung kultureller Sachverhalte für die sozial Interagierenden innerhalb ein und derselben Kommunikations- wie Kulturgemeinschaft als Diasystem kleinerer bis kleinster Teilbereiche verdeutlicht haben. Soziale Interaktion prägt bestimmte kulturelle Verhaltensnormen und kommunikativ-interaktives Handlungsmusterwissen aus, setzt deren gemeinsamen Besitz (möglichst auch die gemeinsame Akzeptanz und Befolgung dieser Regularien) aber zugleich ebenso voraus wie eine möglich große Deckungsbreite hinsichtlich der Bewertung von Sachverhalten, seien diese nun im engeren Sinne kulturelle oder auch nicht.

Der prägende kulturelle Einfluss dürfte nach unserer Überzeugung wohl bspw. dort besonders stark sein, wo das konkrete Interagieren der Menschen, deren Motivation, Ideale, Wertvorstellungen/Einstellungen, etc. im Mittelpunkt des erkennendmitteilenden Interesses stehen, also im weiteren Sinne die Science de l'homme, die Menschenwissenschaften betroffen sind (darunter Soziologie, Politologie, Philosophie, Linguistik, aber auch Biologie, Medizin), weniger jedoch bei naturwissenschaftlichen 
Weltwissenskonzeptualisierungen, wie etwa in der Chemie, Physik, Mathematik, Logik, etc. Das sehr breite und im Hinblick auf den kulturellen Teil von enzyklopädischen Weltwissensrepräsentationen letztlich entscheidende soziokulturelle Hintergrundswissen/ interkulturelle Differential umfasst aber bspw. auch technologisches Wissen, dessen Gefälle wiederum zur Ausprägung von Dominanzen hinsichtlich präferierter Übersetzungsrichtungen (bspw. aus dem Englischen in andere Sprachen und kaum umgekehrt) oder selbst Sprachverwendung (Englisch als lingua franca) führen dürfte. Daneben sei aber auch auf kulturelles Wissen im Sinne von Besitz an Kenntnissen über Werke der Hochkultur, die Produktion solcher kultureller Produkte, aber auch von Institutionen von Bildung und Kultur verwiesen.

3.1 Zugleich müsste aber auch zumindest bereits ansatzweise deutlich geworden sein, dass Kulturelles nicht nur als O’kult und Teil des enzyklopädischen Weltwissens Kognitives prägt, sondern dabei auch - über die enge Interrelation und Interaktion von Kultur - Kognition und Sprache - auch die Sprache als System und als Verwendung sowie die Sprecher in deren Textproduktion und -rezeption beeinflusst. Da Wissen generell, also bspw. auch als O’komm/ O’ling, letztlich intersubjektiv ausschließlich über Versprachlicht-Textuelles validierbar erscheint, scheint es ein legitimes Unterfangen, Kulturdivergenz (wie durch diese erzeugte abweichende Konzeputalisierungen mehr oder minder komplexer Sachverhalte) über eine detaillierte Beobachtung von Texten/Diskursen dingfest zu machen. Dabei wollen wir Kulturelles ebenso wenig wie Kognitives in direkter Weise mit Sprachlichem identifizieren, worauf ja u. a. auch der Umstand hinzuweisen scheint, dass einer LE offenbar in unterschiedlichen Kulturgemeinschaften (vgl. 13) abweichende oder zumindest abweichend konnotierte Sememe.

(13) Vgl. philosophische Bezeichnungen wie Freiheit in der DDR bzw. BRD, aber auch rot oder kommunistisch; aber auch in den Ländern Lateinamerikas und Spaniens - vgl. etwa das in LA verbreitet tabuisierte Verb coger.

3.2 Welche Schlussfolgerungen ergeben sich aus den bisherigen Ausführungen für das Übersetzen, bei dem ja notweniger Weise Sprach- wie Kulturbarrieren überwunden werden bzw. ein Ausweg aus nachstehendem Dilemma gefunden werden muss? Dieses Dilemma manifestiert sich darin, dass sich normalerweise die 
Verstehensvoraussetzungen entweder von Sender/Autor des $\mathrm{T}_{\mathrm{AS}}$ und Translator nicht, wohl aber die von AS-Sender und ZS-Rezipienten als Fachexperten weitgehend decken - von den hier thematisierten soziokulturellen Aspekten einmal abgesehen, d.h. auf eingeschränkte, strikt naturwissenschaftliche Textsortenexemplare bezogen - bzw. umgekehrt die Verstehensvoraussetzungen von AS-Sender und Translator weitgehend übereinstimmen (die entsprechende landeskundlich-historisch-literarische AS-KulturKompetenz vorausgesetzt bzw. über Recherche erworben), letzterer aber sich mehr oder weniger divergierenden, weil in hohem Maße soziokulturell geprägten Verstehensvoraussetzungen der ZS-Rezipienten gegenüber sieht, die er zu kompensieren bestrebt sein muss.

Dabei kann davon ausgegangen werden, dass das soziokulturelle Hintergrundwissen prinzipiell mehr Divergenzen als Konvergenzen erwarten lässt, wofür komplexe historische, soziopolitische, ideologische, sozioökonomische, juristische, moralischethische, aber auch völkerpsychologisch-mentalitätsmäßige Beweggründe angeführt werden können. Selbst über Jahrhunderte bestehende regelmäßige Austauschbeziehungen (so zwischen Frankreich und Deutschland; Spanien und Portugal bzw. Frankreich, etc.) dürften dabei kaum hinreichend ausgleichend, homogenisierend gewirkt haben.

3.2.1 Wie bereits im Zusammenhang mit der gemutmaßten unterschiedlichen Präsenz involvierter kultureller Faktoren, bspw. in naturwissenschaftlichen Texten, aufgezeigt, muss eine detailliertere Bestimmung dessen erfolgen, was an Textsorten mit welcher Thematik, mit welchem Kommunikationsgegenstands- bzw. Weltwissensbezug, übersetzt wird. Immerhin hat schon NEUBERT 1968 darauf hingewiesen, dass wir es beim Übersetzen mit mehreren Arten von Textthemenbezug sowie involvierter Pragmatik zu tun haben mit deutlichen Konsequenzen für die bei der Translation notwendig statthabende Brechung der Pragmatik, d.h. Wechsel in einen anderen soziokulturellen wie generell lebensweltlichen Hintergrund. Man kann dabei davon ausgehen, dass die Pragmatik bei zumindest ausgewählten naturwissenschaftlichen Texten, darunter allerdings keinen mit irgendwie geartetem direktem Bezug auf den Menschen und dessen Interaktion (so bei ökonomischen, biologisch-medizinischen, 
juristischen, neben historisch-soziologisch-philosophischen Texten), gegen Null tendiert, d.h. die kulturelle Prägung ein absolutes Minimum erreicht ${ }^{20}$.

Anders, wenn bspw. ein spezifisch AS-Hintergrund gerichteter Text als Original vorliegt bzw. solche spezifischen AS-Hintergrundwissenstatbestände dominierende Charakteristika des Originals darstellen.

Bei NEUBERT 1968 figurieren letztere als eigentlich nicht zu übersetzende Texte, wobei wohl in der Tat nur in sehr speziellen Ausnahmefällen für ein solches Original überhaupt ein Übersetzungsbedarf und/oder Übersetzungsauftrag vorliegen dürfte. Einen weiteren Sonderfall führt bspw. B. SPILLNER 1992 an, etwa die Zeitungsannonce des Wahlgewinners. Hier führt er eine spezielle Textsorte vor, die AS-spezifisch (Frankreich bezogen und damit eine Art Designatsrealie des soziokulturellen Hintergrunds) ist, d.h. für die es mangels Bezeichnungsnotwendigkeit bzw. fehlendem soziokulturellen Usus in der ZS in Deutschland keine vergleichbaren Textsortenexemplare gibt.

Wir haben es bei einem solchen seltenen Fall also mit einer in gewissem Umfang mit den 1:Nullentsprechungen auf der Lexemebene vergleichbaren Situation auf der Verwendungsebene zu tun. Dabei sind für den referierten Sachverhalt allerdings im Unterschied zur Lage bei tatsächlichen Realienlexemen keine Neukonzeptualisierungen notwendig, sondern müsste allenfalls eine geeignete Textpräsentation vorgeschlagen werden, für die der AS-Text eine durchaus geeignete Bezugsgrundlage selbst im Sinne einer so genannten dokumentarischen Übersetzung (NORD 2002) sein könnte. Zudem ist bei einer solchen Textsorte, die durchaus in hohem Maße AS-spezifisch basiert ist, im Unterschied zu den eingangs erwähnten Fällen oder auch konstruierten Fällen ${ }^{21}$ ein Übersetzungsbedarf oder Auftrag durchaus wahrscheinlicher.

Ebenfalls mit einer von Fall zu Fall kaum weniger dominant AS-spezifisch kulturgeprägten Textsorte haben wir es bei literarischen Texten mit AS-Bezug ${ }^{22}$ zu tun bzw. auch bei feuilletonistischen Texten mit detaillierter Darstellung der ASspezifischen lebensweltlichen Praxis bzw. mit deutlich vorausgesetztem impliziten AS-

\footnotetext{
${ }^{20}$ Das indes nicht gleich Null ist, weil in diesen Texten immerhin ein bestimmtes technologischmethodologisches Wissen reflektiert erscheint, das kulturkreisspezifisch geprägt sein kann; vgl. das technologische Wissensdefizit zwischen Amerika/England und weiteren Ländern.

21 Wir verweisen in diesem Zusammenhang etwa auf theoretisch relevante, aber praktisch undenkbare Fälle, in denen bspw. gefordert würde, einen hoch spezialisierten Fachtext zur Kernphysik in die Sprache eines neu entdeckten Indianerstammes am Amazonas mit steinzeitlicher Kulturstufe zu übersetzen.

${ }^{22}$ Science fiction Werke wie auch Romane mit nicht AS-spezifischer Einbettung wären eine hier nicht anzusprechende Ausnahme.
} 
Vorwissen als geteilter Referenzbasis für die Erschließung des Mitverstandenen. Hier treten bei der Übersetzung von im Einzelnen deutlich abweichenden Sachverhalten, Verhaltensweisen, Einstellungen, etc. in AS- und ZS-Kultur mit einer Vielzahl spezieller, keineswegs leicht zu bewältigender Probleme zu tun, für die u. W. die translatologische Forschung bislang noch kein Patentrezept vorweisen kann. Denken wir nur an eine im AS-Text deutlich als deviant markierte Verhaltensweise, die im Hinblick auf die abweichende ZS-Kultur eine angemessene, prototypische Verhaltensweise wäre oder auch umgekehrt. Wie entscheidet sich der Übersetzer hier? Dabei steht er in solchen und zahlreichen weiteren Fällen vor der selbst gefällten oder auch vor der ihm durch die Skoposfestsetzung, den Übersetzungsauftrag, vorgegebenen Entscheidung, bspw. für die ZS-Textproduktion die ZS-Kultur als dominanten Richtwert zu betrachten oder aber bewusst das Risiko einzugehen, dass bei der unvermeidbaren Brechung der Pragmatik ${ }^{23}$ so manche im $\mathrm{T}_{\mathrm{AS}}$ getroffene Aussage und dargelegte normkonforme Verhaltensweise dem ZS-Rezipienten ungewöhnlich, kritikabel, intolerabel vorkommt bzw. sich diesen die im AS-Text enthaltenen Anspielungen erst durch eine umfängliche vertextete Rekonstruktion des fehlenden Verstehenshintergrundes nachvollziehbar werden.

3.2.2 Bislang hat die Translatologie u. E. ungeachtet verstärkter Bemühungen um empirische Analysen (vgl. TOURY 1995) noch nicht im Einzelnen hinreichend erhellen können, welche Strategie der Translator literarischer Texte nun wirklich verfolgen soll, ob er bspw. eine Nachgestaltung der AS-Kultur nicht nur als unvermeidliches Übel, sondern als Desiderat betrachten soll, wohl bewusst der Tatsache, dass damit Anstrengungen seitens des Lesers verlangt werden, sich dieser ihm fremden Kultur zu öffnen. Ein solches translatorisches Credo würde ihm die Entscheidung von Fall zu Fall leichter machen, stünde aber im Gegensatz zu anderen literarisch-kulturtheoretischen Vorgaben und richtet sich zudem diametral gegen das bspw. für die Übersetzung von Fachtexten, insbesondere aber von technischen Gebrauchstexten wie etwa Gebrauchs/Bedienungsanleitungen oder auch Beipackzettel, nachdrücklich und kompromisslos geforderte, möglichst totale Anpassen an die Konventionen der ZS. Im Sinne einer konsequent skoposzentrierten funktionalen Übersetzungstheorie (vgl. besonders VERMEER 1996) sollte die ZS-Version sich faktisch in nichts von einem ohne

${ }^{23}$ Ihre ZS-Kultur können die ZS-Rezipienten nicht ablegen noch die ersatzweise AS-Kultur anziehen oder 
Rekurs auf eine AS-Informationsvorlage, ein Informationsangebot, produzierten ZSText unterscheiden. Allerdings muss man sich in diesem Zusammenhang fragen, ob eine solche Vorgehensweise bei deutlicher AS-Kultur gerichteten Texten, wie etwa auch den literarischen, überhaupt machbar oder sinnvoll wäre, ginge dabei doch eine ganz entscheidende Zweckbestimmung/Funktion solcher Texte verloren. Dabei schiene es im Prinzip ein interessantes Gedankenexperiment, dass bspw. die gesamte, in den AS-Hintergrund eingebettete Handlung, einschließlich deren konkreter Lokalisierung, also im Text referierten geographisch-klimatischen Umfeldbedingungen, samt und sonders in einen ZS-Hintergrund verpflanzt würde. Immerhin würde dies enorme Veränderungen erfordern und möglicherweise dort an Grenzen stoßen, wo etwa klimatische Bedingungen kommunikativ relevant und selbsterklärend für das Verständnis des Handlungsverlaufes und/oder das Verhalten von Personen sind ${ }^{24}$.

Theoretisch würde so eine AS-Diskurswelt in toto oder auch nur in Teilen komplett durch eine ZS-Lebens- und Diskurswelt ersetzt. Würde damit aber nicht das von uns nach wie vor als konstitutiv betrachtete Prinzip der weitestgehenden Aufrechterhaltung der kommunikativen Äquivalenz über Bord geworfen? Schiene eine solche Vorgehensweise durch eine wünschenswerte Hypostasierung der ZS-Kultur als Referenzpunkt für adäquates translatorisches Handeln gerechtfertigt? Übersetzen verbleibt für uns - bei aller Beachtung der kommunikativen Akzeptabilität, der Angemessenheit im Hinblick auf den sprachlichen USUS, die Bezeichnungs- wie Textsortengestaltungsgepflogenheiten in makro- wie mikrostruktureller Hinsicht immer eine semantisch-referentiell wie konnotativ der AS-Botschaft verpflichtete Tätigkeit in dem Sinne, dass ein dem kommunikativen Sinn, dem intendierten bzw. realisierten kommunikativen Effekt des $\mathrm{T}_{\mathrm{AS}}$ weitgehend deckungsgleicher, kommunikativ äquivalenter $\mathrm{T}_{\mathrm{ZS}}$ produziert werden sollte, der zudem auch den entsprechenden ZS-Textsortennormen gerecht wird und übliche Bezeichnungsstereotype (vgl. 14 a und b) berücksichtigt.

(14) a) craint la pluie gegenüber ne pas exposer à l'humidité; défense de fumer (et de cracher); hay perro - Vorsicht vor dem Hunde; aquí se da pie todavía - hier kann man noch stehen; Verkehrsschild "Bauarbeiten" - in Spanien "Obras" - in Kuba "Hombres trabajando"; no pisar el césped; an Türen empuje/tire; è pericoloso sporgersi -

auch nur überziehen wie einen Mantel.

${ }^{24}$ Wenn jemanden bspw. die ständige Sonne ausdörrt und er sich nach Regen sehnt; hier wäre dies gegebenenfalls dahingehend zu transponieren, dass jemanden der ständige Regen auf die Nerven geht und er sich nach der Sonne sehnt. 
Hinauslehnen verboten!; señores viajeros al tren - bitte einsteigen! Betteln und Hausieren verboten, etc.

b) lexikalisch in Komposita manifestierte Konzeptualisierungsunter- schiede innersprachlich/interlingual:

danger de mort/peligro de muerte - Lebensgefahr; Schraubenzieher

Schraubendreher; Zollstock $\rightarrow$ Metermaß $\rightarrow$ Gliedermaßstab; Oficina de objetos perdidos - Bureau des objets trouvés/Fundbüro; Mi tía se fue muy adentro al mar y le costó volver a la costa, etc..

Dass dabei realiter wohl nie eine wirklich hundertprozentige Deckungsgleichheit in jeder Hinsicht möglich ist, ist einleuchtend; es kann also in der Regel nur darum gehen, einen ZS-Text zu produzieren, der möglichst weitgehend deckungsgleich ist, wobei der Grad an Äquivalenz ${ }^{25}$ nicht leicht zu bestimmen und vor allem der Punkt, bei dem die Heterovalenz an Übergewicht gewinnt, sehr schwer feststellbar ist. Uns scheint aber theoretisch wie praktisch klar, dass bei funktionsvarianter Übersetzung zumeist ein heterovalenter Text produziert wird, dem wir die Qualität einer Übersetzung absprechen und von ihm als einer Adaptation sprechen würden ${ }^{26}$. Wir sind uns bewusst, dass wir uns damit im Widerspruch zur vorherrschenden Meinung befinden, dass auch solche Übertragungen als Übersetzungen im weiteren Sinne zu bezeichnen wären. Selbst wenn in einem terminologisch stringenten Sinne dann besser nicht von Übersetzung gesprochen werden sollte, so wäre ein solcher Auftrag dennoch dem Arbeitsprofil von Übersetzern zuzurechnen und in der Praxis nicht unüblich. In eine letztlich ähnliche Richtung zielen Auffassungen, dass alle die ZS-Texte als Übersetzungen zu betrachten seien, die aus einer Übertragung aus einem AS-Text entstanden seien, ungeachtet einer Überprüfung, ob zumindest ein Mindestmaß an äquivalenten Textteilen nachgewiesen werden kann und ob bspw. das Gemeinte weitgehend realisiert wird.

\section{Resümierend möchten wir festhalten:}

4.1 Übersetzen ist eine eminent kognitive Tätigkeit, wobei zwischen Kognition und Kommunikation nicht nur kein Gegensatz, sondern vielmehr eine unauflösbare Einheit besteht.

4.2 Beim Übersetzen muss davon ausgegangen werden, dass sowohl Kognition wie auch Kommunikation und darunter besonders Sprache in mannigfacher Weise kulturgeprägt bzw. mit Kulturellem verbunden sind.

\footnotetext{
${ }^{25}$ Was nicht Identität, sondern Gleichwertigkeit bedeutet; Näheres dazu vor allem in WOTJAK 1995, 1997a; 2003, 2007.

${ }^{26}$ Mit Nutzen sollten dazu die subtilen Ausführungen von M. SCHREIBER 1993 eingesehen werden.
} 
4.3 Nach übereinstimmender Meinung dürfte stets ein mehr oder minder großes Differential zwischen den soziokulturellen, lebensweltlichen Umfeldbedingungen der Menschen in geographisch-klimatisch wie historisch-politisch-ökonomisch-ideologisch spezifisch geprägten realen wie mentalen Räumen nachweisbar sein. Dieses soziokulturelle Differential als umfassend-generische Größe manifestiert sich direkt als (para-, aber auch als dia-) kulturelles Wissen O'kult anteilig eingespeichert in den lexikalischen Bedeutungen ausgewählter lexikalischer Indikatoren (hervorstechendes Beispiel die Realienlexeme), aber auch in geteilten kulturgeprägten Weltwissensrepräsentationen (Szenen, Szenarien, kulturellen Räumen/Schemata, etc.), als Handlungsmusterwissen wie Verhaltensstereotypkenntnis, wobei mehr oder minder umfängliche und komplexe Sachverhaltswissenszusammenhänge als Hintergrundswissen über LE-Bedeutungen indiziert bzw. induziert (dazu FILLMORE 1985/86; WOTJAK 1991a; 2006a) wird.

4.4 Kuturelles manifestiert sich darüber hinaus aber auch in Präferenzen für die Selektion, Anordnung und Kombination von sprachlichen Systemelementen lexikalischer wie morphosyntaktischer Art zu Textsortenexemplaren unter Beachtung kulturgeprägter Bevorzugung bestimmter Sprechakte und Kommunikationsstrategien, aber bspw. auch für die Anordnung von Informationsblöcken, die Proportion von Explizitem/ Gesagtem und Impliztem/Mitverstandenem, von sprachlichen und weiteren semiotischen Mitteln im Text sowie von bestimmten Präsentationsformen von Wissen im Sinne von writer bzw. reader-responsiver Schreibweise.

4.5 Beim Übersetzen kommt als übergeordnetes Regulativ das jeweilige translatorische Credo zum Tragen, das dem Translator als Richtschnur für sein translatorisches Handeln dient. Dabei besitzt für das Übersetzen zweifellos die in der Leipziger Schule nur allzu generisch behandelte bzw. weitgehend übergangene Skoposvorgabe durch den Auftraggeber ein besonderes Gewicht, nimmt sie dem Translator im günstigsten Fall doch die Qual der Wahl ab hinsichtlich der Verpflichtung zur Treue/Loyalität gegenüber dem Original, d.h. zur Erzeugung eines kommunikativ äquivalenten ZS-Textes bzw. auch zur Produktion eines heterovalenten ZS-Textes im Ergebnis einer ausdrücklich erbetenen funktionsvarianten Übertragung.

Dabei hat der Translator unseres Erachtens bei geforderter, aber auch bei nicht ausdrücklich untersagter funktionskonstanter Übertragung (die u. E. in der Übersetzungspraxis dominant ist) die Pflicht zur Anfertigung einer der AS-Textwelt 
weitgehend kommunikativ äquivalenten ZS-Textwelt, was angesichts des Vorhandenseins von Divergenzen in den Verstehensvoraussetzungen zwischen $\mathrm{T}_{\mathrm{AS}}$ - und

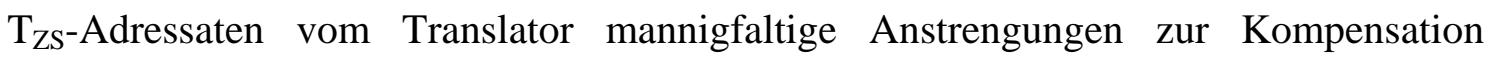
ermittelter/ gemutmaßter Verstehensdefizite hinsichtlich einer vollen Sinnerfüllung voraussetzt.

4.6 Unter Berücksichtigung des soziokulturellen Differentials erfordert die Übersetzung eines spezifisch AS-gerichteten Originals eine besonders hohe translatorische Meisterschaft, naturwissenschaftlich-mathematische Texte dagegen erweisen sich als deutlich weniger kulturgeprägt divergierend, weil bspw. diakulturell kulturkreisübergreifend weitgehend übereinstimmend. Hier verfügen bei fachinterner Expertenkommunikation AS- wie ZS-Rezipienten über annähernd gleiche Verstehensvoraussetzungen, während diese beim Translator dagegen mitunter fehlen und erst mittels Recherche erworben werden müssen. Auch in diesen Texten spielt die Beachtung der kommunikativen Angemessenheit eine große Rolle.

4.7 Wiewohl auch die literarische Übersetzung gewissen Textsortenkonventionen verpflichtet sein dürfte, ist deren Verbindlichkeit deutlich geringer und die Gestaltungsmöglichkeit letztlich unbeschränkt frei. Zudem ist der Translator hier im Allgemeinen gehalten, sowohl den Stil und die Gedanken als auch die Empfindungswelt des Autors und der handelnden Personen möglichst getreu in die ZS zu transponieren/ traducere navem ${ }^{27}$. Die durch den Übersetzer $\mathrm{zu}$ beachtende kommunikative Äquivalenz und kommunikative Angemessenheit schließen einander nicht aus, sondern ergänzen einander, wobei je nach Textsorte und Festlegung der dominanten Übersetzungsstrategie (instrumentell oder dokumentarisch) die Angemessenheit gegenüber der u. E. deutlich dominierenden kommunikativen Äquivalenz einen größeren oder kleineren Raum einnimmt.

Insbesondere technische Gebrauchstexte, wie Bedienungsanleitungen für Autos, Staubsauger, etc. oder auch Beipackzettel für Arzneien (KuLOW 1999) sollen sich lesen wie ZS-Originaltexte und den dafür gültigen makro- wie mikrostrukturellen Textsortenformulierungsregeln gerecht werden, was die Paralleltextanalyse noch wertvoller erscheinen lässt und bis hin zur Einfügung von zusätzlichen Teiltexten bzw.

\footnotetext{
${ }^{27}$ Zur Problematik, die mit den unterschiedlichen (metaphorischen) Bestimmungen des Übersetzens als zweisprachig vermittelte Kommunikation, als Kulturtransfer, als Brückenbau (HöNIG 1995), als Übersetzen an andere Ufer (Grimm), Umkodierung (KADE 1968), etc. verknüpft sind - vgl. u. a. C. MARTÍN DE LEÓN 2005.
} 
zu deren Eliminierung führen kann im Einklang mit den gesetzgeberischen Vorschriften, die bspw. zwischen Deutschland und Frankreich signifikant abweichen.

4.8 Übersetzen führt als kreative ZS-Textgestaltung unter Beachtung der AS-Textwelt und Verstehensvoraussetzungen der ZS-Rezipienten sowie der gegebenen Textproduktions- wie -rezeptionsbedingungen selbst bei weitgehender Einlösung des Anspruchs auf Treue bzw. Loyalität keineswegs automatisch zu nur einer optimalen Version. Vielmehr entstehen auch bei identischer Skopossetzung in der Regel verschiedene, untereinander mannigfaltig abweichende und dennoch gleichermaßen als optimal zu bezeichnende Translate. Das allerdings bedeutet keinen Freibrief, keinen Verzicht auf eine möglichst gelungene Äquivalentfindung. Immerhin muss beachtet werden, dass u. U. semantisch - denotativ wie konnotativ völlig korrekte Übersetzungsäquivalente durchaus nicht auch als tatsächliche Entsprechungen fungieren, weil sie eben nicht kommunikativ angemessen sind, dem vorherrschenden Usus der ZS nicht entsprechen (vgl. die unter 14a erwähnten Beispiele). Der Translator ist eben gehalten, nicht nur das Gesagte zu beachten, sondern es auch gemäß den Konventionen der ZS zu formulieren, d.h. gegebenenfalls auf formelhafte Ausdrücke zu rekurrieren. Außerdem muss er versuchen, das Mitverstandene als Teil der Mitteilung und AS-Diskurswelt vor dem Hintergrund abweichender Vorwissenstatbestände der ZSRezipienten zu bewahren, was ihn bspw. zu Vertextungen veranlasst, wodurch die Relation von Explizitem und Implizitem verschoben wird. Schließlich muss der Translator zu gewährleisten versuchen, dass die Kommunikationsabsicht wie der übergeordnete Handlungszweck des AS-Diskurses im ZS-Diskurs gewahrt werden, von sonstigen Aspekten wie der Wahrung der Stilebene/ Stilfärbung, etc. ganz abgesehen.

4.9 Dass die Übersetzungen ungeachtet weit reichender Beachtung von Akzeptabilität/ Ususangemessenheit in der Regel eine eigene Textsorte sui generis in der ZS konstituieren, ist bereits von verschiedener Seite herausgestellt worden. Dass sie als Brückenschläge nicht zuletzt auch der dank der über diese Brücken transportierten Ausgangskulturwissenskomponenten auch zur Ausweitung des Fremdkulturwissens beitragen und damit diesbezügliche Vorwissensdefizite erfolgreich auszugleichen ermöglichen, hat positive Konsequenzen für nachfolgende Übersetzungsaktivitäten, die auf diesem übermittelten AS-Kulturwissen in der ZS-Gemeinschaft aufbauen können. Transferre necesse est - und dies nicht nur aus unmittelbar ökonomischen oder kulturellen Zwängen, sondern letztlich auch, weil nur so zu einer in der 
zusammenwachsenden Welt dringend wünschenswerten umfassenderen Verständigung über Kultur- und Sprachgrenzen hinaus beigetragen werden kann.

\section{Literaturverzeichnis:}

Bastian, Sabine. Die Rolle der Präinformation bei der Analyse publizistischer und belletristischer Texte im Französischen und Deutschen. Diss., Univ. Leipzig. 1974.

BASTIAN, Sabine: Die Rolle der Präinformation bei der Analyse und Übersetzung von Texten. In: Übersetzungswissenschaftliche Beiträge, H.2., Leipzig, Enzyklopädie, 1979, 90-133.

BIERWISCH, Manfred. Das Organ des Denkens und die Grenzen des Ausdrückbaren. In: Werkzeug Sprache. Sprachpolitik, Sprachfähigkeit, Sprache und Macht. (3. Symposium der deutschen Akademien der Wissenschaften). Hildesheim/ Zürich/ New York: Olms, 1999, 57-101.

BÜHLER, Karl. Sprachtheorie. Jena, 1934.

Campos Plaza, /Ortega Arjonilla, E. Panorama de Lingüística y Traductología. Atrio, Ediciones de la Universidad de Castilla-La Mancha, 2005.

CARY, Édmond. La traduction dans le monde. Genève, 1960.

CATFord, John C. A linguistic theory of translation. An essay in applied linguistics. London: Oxford University Press, 1965.

CLYNE, Michael. Cultural differences in the organization of academic texts. In: Journal of Pragmatics, $\mathrm{N}^{\circ}$ 11, 1987, 211-247.

COSERIU, Eugenio. Lo erróneo y lo acertado en la teoría de la traducción. In: El hombre y su lenguaje: Estudios de teoría y metodología lingüística, Madrid: Gredos, 1977, 214-239.

COSERIU, Eugenio. Einführung in die allgemeine Sprachwissenschaft. Tübingen: Narr, 1988.

Ducrot, Oswald. Dire et ne pas dire. Principes de sémantique linguistique. Paris: Herman, 1972.

FERnÁNDEZ Polo, Francisco Javier. Traducción y retórcia contrastiva. A propósito de la traducción de textos de divulgación científica del inglés al español, Universidade de Santiago de Compostela: Servicio de publicaciones, (=Colección Lucus-Lingua, 6), 1999.

FILLMORE, Charles J. Frame Semantics and the Nature of Language. In: Annals of the New York Academy of Science, 280. New York, 1976, 20-31. 
FleischmanN, Eberhard. Translatorische Kulturkompetenz als Wissensphänomen. In: EmSel, M. / Hellfayer, A. (eds.): Brückenschlag. Beiträge zur Romanistik und Translatologie. Gerd Wotjak zum 60. Geburtstag. Frankfurt et al.: Peter Lang, 2003, 173-194.

FRIEDRICH, Wolfgang. Technik des Übersetzens: Englisch-Deutsch. München: Hueber, 1977.

GALTUNG, Johan. Struktur, Kultur und intellektueller Stil. Ein vergleichender Essay über sachsonische, teutonische, gallische und nipponische Wissenschaft. In: Levithan. Zeitschrift für Sozialwissenschaft, Nr. 11, 1983, 303-338.

Gerzymisch-Arbogast, Heidrun. Contrastive scientific and technical register as a translation problem. In: Susan WRIGHT/Leonard WRIGHT (eds.): Scientific and technical translation. Amsterdam/ Philadelphia, Benjamins, 1993, 21-51.

Gerzymisch-ArbogAst, Heidrun/ Mudersbach, Klaus. Methoden des wissenschaftlichen Übersetzens. Tübingen: Stauffenburg, 1997.

Grosse, Rudolf/NEUBERT, Albrecht. Thesen zur marxistisch-lenenistischen Soziolinguistik. In: Beiträge zur Soziolinguistik. Leipzig: Enzyklopädie, 1974, 25-46.

HAuser, Gerlind. Ein Kulturmodell für Translatoren. In: SchMitT, Peter A./JÜNGST, Heike (eds.): Translationsqualität. Peter Lang (Leipziger Studien zur angewandten Linguistik und Translatologie, 5) auf der beigefügten CD, 2007.

HINDS, John. Reader versus writer responsibility: a new typology. In Ulla ConNOR \& Richard KAPLAN (eds.): Writing across languages: Analyses of L2 text, Reading (Mass.), 1987, 141-152.

HÖNIG, Hans. Konstruktives Übersetzen. Tübingen: Stauffenburg, 1995.

HOLTZ-MÄNTTÄRI, Justa. Translatorisches Handeln. Theorie und Methode. Annales Academiae Scientiarum Fennicae B 226. Helsinki: Suomalainen Tiedeakatemia, 1984.

HuRTAdo AlbiR, Amparo. Traducción y Traductología. Introducción a la Traductología. Madrid: Cátedra, 2001.

JÄGER, Gert. Translation und Translationslinguistik. Halle: Bibliographisches Institut, 1975.

JÄGER, Gert/MÜLlER, Dietrich. Kommunikative und maximale Äquivalenz. In: Übersetzungswissenschaftliche Beiträge, V. Leipzig, Enzyklopädie, 1982, 4257. 
JÄGER, Gert. Die sprachliche Bedeutung - das zentrale Problem bei der Translation und ihrer wissenschaftlichen Beschreibung. In: Übersetzungswissenschaftliche Beiträge IX. Leipzig: Verlag Enzyklopädie, 1986, 5-66.

JunG, Linus. La Escuela Traductológica de Leipzig. Granada: Ediciones Colmares, 2000.

KADE, Otto. Zufall und Gesetzmäßigkeit in der Übersetzung. Beiheft I zu Fremdsprachen. Leipzig: Enzyklopädie, 1968.

KADE, Otto. Das Problem der Übersetzbarkeit aus der Sicht der marxistischleninistischen Erkenntnistheorie. In: Linguistische Arbeiten/LAB 4. Leipzig, 1971, 13-28.

KLAUS, Georg. Die Macht des Wortes. Berlin, 1964.

KOLLER, Werner. Einführung in die Übersetzungswissenschaft. Heidelberg: Quelle. ${ }^{4} 1992$.

KRINGS, Hans-Peter. Was in den Köpfen von Übersetzern vorgeht. Eine empirische Untersuchung zur Struktur des Übersetzungsprozesses an fortgeschrittenen Französischlernern. Tübingen: Narr, 1986.

KulOw, Gesine. Zu einem Vergleich ausgewählter medizinischer Texte am Beispiel von Packungsbeilagen für Medikamente Französisch-Deutsch. Diplomarbeit am Institut für Angewandte Linguistik und Translatologie der Universität Leipzig, 1999.

LEDERER, Marianne. La traduction aujourd'hui. Le modèle interprétatif. Paris: Minard, 1994.

LEFEVERE, André. Traducción, reescritura y la manipulación del canon literario. Salamanca: Ediciones Colegio de España, 1996.

LERCH, Eugen. Französische Sprache und Wesensart. Frankfurt a. M.: Verlag Moritz Diesterweg, 1933.

LORENZ, Wolfgang/WotJAK, Gerd. Zum Verhältnis von Abbild und Bedeutung. Berlin: Akademie-Verlag, 1977.

Malblanc, Albert. Stylistique comparée du français et de l'allemand. Paris. 1968.

MARTín DE LEÓn, Celia. Contenedores, recorridos y metas. Metáforas en la traductología funcionalista. Frankfurt et al.: Peter Lang (Reihe "Studien zur romanischen Sprachwissenschaft und interkulturellen Kommunikation“, vol. 24), 2005.

Martín Morillas, J. M. / PéRez Rull, J. C. Semántica cognitiva intercultural. Granada: Univ. (serie Granada Lingvistica), 1998. 
MontaÑo-Harmon, Miguel R. Discourse features of written Mexican Spanish: Current research in contrastive rhetoric and its implications. In: Hispania 74, 1991, 417425.

Mounin, George. Les problèmes théoriques de la traduction. Paris, 1963.

Neubert, Albrecht. Pragmatische Aspekte der Übersetzung. In: Fremdsprachen, Beiheft II. Leipzig: Enzyklopädie, 1968, 21-33.

NeUBERT, Albrecht. Text and Translation, Übersetzungswissenschaftliche Beiträge 8. Leipzig: Enzyklopädie, 1985

Neubert, A. / Shreve, G. M. Translation as Text. Kent Studies in Translation 1. Kent, Ohio, London, 1992.

NIDA, Eugene A. Toward a Theory of Translating. Leiden: E. J. Brill, 1964.

NORD, Britta. Eine empirische Studie zum Rechercheverhalten professioneller Übersetzer. Frankfurt et al.: Peter Lang, 2002.

NoRD, Christiane. Textanalyse und Übersetzen. Heidelberg: Groos, 1988.

NoRD, Christiane. Kommunikativ handeln auf Spanisch und Deutsch. Übersetzungsorientierte komparative Stilistik. Wilhelmsfeld: Gottfried Egert Verlag, 2002a.

NoRD, C. Fertigkeit Übersetzen. Alicante: Editorial Club universitario, 2002b.

OKSAAR, Els. Kulturemtheorie. Ein Beitrag zur Sprachverwendungsforschung. Hamburg, Burske, 1988.

Ortega ArJonilla, Emilio. Apuntes para una teoría hermenéutica de la traducción. Universidad de Málaga: Colección Estudios y Ensayos. 1996.

Ortega ArJonilla, Emilio. Filosofía, traducción y cultura. In: Román Álvarez (ed.): Cartografía de la traducción. Del post-estructuralismo al multiculturalismo. Salamanca: Ediciones Almar. Biblioteca de la Traducción, 2001, 177-214.

Ortega ARJONiLla, Emilio. La traducción como actividad mediada culturalmente: revisión crítica de la tesis de la indeterminación de la traducción de Quine. In: GARCíA Marcos, P. et alii (eds.). Traducción, cultura e inmigración. Reflexiones interdisciplinares. Granada: Atrio, 2004, 61-76.

REIß, Katharina. Möglichkeiten und Grenzen der Übersetzungskritik: Kategorien und Kriterien für eine sachgerechte Beurteilung von Übersetzungen. München: Hueber, 1971.

REIß, Katharina \& VermeER, Hans J. Fundamentos para una teoría funcional de la traducción. Madrid: Akal, 1996. 
RISKU, Hanna. Translatorische Kompetenz. Tübingen: Stauffenburg, 1998.

SÁEz Hermosilla, Teodoro. El sentido de la traducción: reflexión y crítica. Univ. de León: Secretariado de Publicaciones, Universidad de Salamanca. 1994.

SALEVSKy, Heidemarie. Translationswissenschaft. Ein Kompendium. vol.1. Frankfurt et al.: Peter Lang, 2002.

SchmitT, Peter A. Translation und Technik. Tübingen: Stauffenburg, 1999.

SCHREIBER, M. Übersetzung und Bearbeitung: Differenzierung und Abgrenzung des Übersetzungsbegriffes. Tübingen: Narr, 1993.

SEleskovitch, Danica/ Lederer, Marianne. Interpréter pour traduire. Col. Traductologie, 1. Paris: Didier Érudition, 1984.

SIEVER, Holger. Übersetzen und Interpretation. Die Herausbildung der Übersetzungswissenschaft als eigenständige Disziplin im deutschen Sprachraum von 1960 bis 2000. Habilitationsschrift eingereicht an der Universität Leipzig (Ms.), 2008.

Snell-Hornby, Mary. Translation Studies - An integrated approach. Amsterdam / Philadelphia: Benjamins, 1988.

Snell-Hornby, M. / PöchHACKer, F. / Kaindl, F. (eds.). Translation Studies: An Interdiscipline. Amsterdam/Philadelphia: Benjamins, 1994.

Snell-Hornby, M. / Hönig, H. / KußMaul, P. / SchmitT, P.A. (eds.) Handbuch der Translation. Tübingen: Stauffenburg, 1998.

SPILlNER, Bernd. Probleme des deutsch-französischen Sprachvergleichs im Hinblick auf die Landeskunde. In: französisch heute, 23. Jahrgang, no. 2, 1992, 388-397.

SPILlnER, B. Terminologie et connotations. In: CANDEL, D. (ed.) Français scientifique et technique et dictionnaire de langue. Paris, 1994, 53-62.

STEDJE, Astrit. Beherztes Eingreifen oder ungebetenes Sich-Einmischen. Kontrastive Studien zu einer ethnolinguistischen Phraseologieforschung. In: GRÉCIANO, G. (ed.): Europhras 88. Phraséologie contrastive. Strasbourg: Université (=Collection Recherches Germaniques, 2), 1989, 441-452.

TOURY, Gideon. Descriptive Translation Studies-and beyond. Amsterdam: John Benjamins, 1995.

VERMEER, Hans J. A skopos theory of translation. (Some arguments for and against). Heidelberg: Groos, 1996.

VidAl Claramonte, Carmen A. Traducción, manipulación, deconstrucción. Salamanca: Ediciones Colegio de España, 1995. 
Vidal Claramonte, Carmen A. El futuro de la traducción. Últimas teorías, nuevas aplicaciones. Valencia: Institució Alfons el Magnànim, 1998.

WANDRUSZKA, M. Sprachen vergleichbar und unvergleichlich. München: Piper, 1969.

WeISGERBer, L. Vom Weltbild der deutschen Sprache. Düsseldorf: Schwann, 1950.

WiTTE, Heidrun. Die Kulturkompetenz des Translators. Begriffliche Grundlegung und Didaktisierung. Tübingen: Stauffenburg, 2000.

WotJAK, Gerd. Illokution und Perlokution in translationslinguistischer Sicht. In: Leipziger Arbeitsberichte. 47. Leipzig, 1985, 38 - 48.

WотנAK, Gerd. $\mathrm{Zu}$ den Interrelationen von Bedeutung, Mitteilungsgehalt, kommunikativem Sinn und kommunikativem Wert. In: Übersetzungswissenschaftliche Beiträge, IX. Leipzig, 1986, 67 - 127.

WотנAK, Gerd. Kommunikatives Wissen in interlingualer und interkultureller Sicht. In: ZPSK, vol. 44, 1 (1991). Berlin, 1991a, 111 - 125.

WotJAK, Gerd. Zum kommunikativen Potential lexikalischer Einheiten. In: Deutsch als Fremdsprache , H. 1, Leipzig, 1991b, 3 - 10.

WotJAK, Gerd. Interkulturelles Wissen und Übersetzen. In: Revista de Filología Germánica no. 1. Madrid: Universidad Complutense, 1993a, 181-196.

WotJAK, Gerd. Interkulturelles Wissen und Sprachvergleich. In: G. Rovere/ G. WOTJAK (Hgg.): Studien zum romanisch-deutschen Sprachvergleich. Tübingen (Linguistische Arbeiten 297), 1993b, 55-68.

WoTJAK, Gerd. Soziokulturelles in der kubanischen Umgangssprache. In: Ibero Americana 54, Nr. 2. 18. Jg. Frankfurt: Peter Lang, 1994, 16-35.

WoTJAK, Gerd. Equivalencia semántica, equivalencia comunicativa y equivalencia translémica. In: Hieronymus, No. 1. Madrid (Universidad Complutense), 1995, 93-112.

WotJAK, Gerd. Äquivalenz und kein Ende? Nochmals zur semantischen und kommunikativen/ translatorischen Äquivalenz. In: G. WoTJAK/ H. SCHMIDT (Hgg.) Modelle der Translation/ Models of Translation. Festschrift zum 65. Geburtstag von Albrecht Neubert. Frankfurt: Vervuert-Verlag, 1997a, 133-170.

WotJAK, Gerd. Bedeutung und Kognition. In: HoInKes, U. /DiETRICH, Wolf (eds.): Kaleidoskop der lexikalischen Semantik. Tübingen: Narr (TBL 428), 1997b, 31-62.

WotJAK, Gerd. ¿Qué abarca la competencia traslatoria? En: El traductor profesional ante el próximo milenio (Actas de las II Jornadas sobre la formación y profesión del traductor-intérprete, Universidad Europea-CEES, 17.2.-20.2.1999). CDRom; file://DI//12.htm. 2001 
WotJAK, Gerd. Sinngebung, Sinndeutung, kommunikativer Sinn, Funktion und Skopos: Sinniges, Widersinniges und Unsinniges im translatologischen Blätterwald? In: B. NoRD/ P.A. SchMiTT (Hgg.) Traducta Navis. Festschrift zum 60. Geburtstag von Christiane Nord. Tübingen: Stauffenburg, 2003, 271- 297.

WotJAK, Gerd. ¿De qué tipos de conocimientos debería disponer el traductor? In: José Gerardo MARTínez DEL CASTILlo (ed.): Eugenio Coseriu in memoriam II. Granada Lingvistica, Granada, 2005a, 123- 141.

WoTJAK, Gerd. Le traducteur à la recherche du sens communicatif de l'original. In: J.PEETERS (ed.) On the Relationships between Translation Theory and Translation Practice. Frankfurt et al.: Peter Lang (Studien zur romanischen Sprachwissenschaft und interkulturellen Kommunikation, 19), 2005b, 53-78.

WotJAK, Gerd. Las lenguas, ventanas que dan al mundo. Universidad de Salamanca: Servicio de Publicaciones, 2006a.

WотJAK, Gerd. La traducción como comunicación bilingüe transcultural mediada. In: HIKMA. 5/2006. Universidad de Córdoba, 2006b, 221-254.

WotJAK, Gerd. Loyalität/ Treue im Hinblick worauf? In: Revista de Filología 25. Universidad de La Laguna, 2007, 629-639.

Zybatow, Lew. Sprache - Kultur - Translation oder Wieso hat Translation etwas mit Sprache zu tun? In: ZyBatow, Lew (Hg.): Translation zwischen Theorie und Praxis. Innsbrucker translationswissenschaftliche Ringvorlesungen I (= Forum Translationswissenschaft, Bd. I). Frankfurt/Berlin/Bern/Bruxelles/New York/Oxford/Wien: Peter Lang, 2002, 61-82. 